\title{
Selected Compounds Modulate Various Inflammatory Biomarkers in Lipopolysaccharide-Induced Macrophages of PPAR- $\alpha$ Knockout Mice
}

\author{
Qureshi $\mathrm{AA}^{* 1}$, Reis JC ${ }^{1,2}$, Badr $\mathrm{MZ}^{2}$, Silswal N${ }^{1}$, and Qureshi $\mathrm{N}^{1,2}$
}

${ }^{1}$ Department of Basic Medical Science, School of Medicine, University of Missouri-Kansas City, Kansas City, USA

${ }^{2}$ Division of Pharmacology and Toxicology, School of Pharmacy, University of Missouri-Kansas City, Kansas City, USA

${ }^{*}$ Corresponding author: Qureshi AA, Department of Basic Medical Science, School of Medicine, University of Missouri-Kansas City, Kansas City, USA, Tel: 816-235-5789, Fax: 816-235-6444, E-mail: qureshia@umkc.edu

Citation: Qureshi AA, Reis JC, Badr MZ, Silswal N, Qureshi N (2017) Selected Compounds Modulate Various Inflammatory Biomarkers in Lipopolysaccharide-Induced Macrophages of PPAR-a Knockout Mice. J Clin Exp Res Cardiol 3(1): 103

Received Date: February 27, 2017 Accepted Date: April 08, 2017 Published Date: April 10, 2017

\begin{abstract}
Background: Inflammation has been implicated in cancer, diabetes and cardiovascular disease. We have recently screened several compounds that modulate inflammatory biomarkers (TNF- $\alpha$, IL- $1 \beta$, IL- 6 , and nitric oxide) in response to a variety of stimuli. Our hypothesis is that compounds with those anti-inflammatory properties will be useful for treatment of diabetes, cardiovascular disease, and other diseases based on inflammation.

Objectives: Present study evaluated 32 compounds of different chemical structures on TNF- $\alpha$ secretion, nitric oxide production, and gene expression of TNF- $\alpha$, IL-1 $\beta$, IL- 6 and iNOS activity in lipopolysaccharide-induced thioglycolate (TG)-elicited peritoneal macrophages from peroxisome proliferator-activated receptor- $\alpha$ (PPAR- $\alpha$ ) knockout mice (that have prolonged response to inflammatory stimuli).

Results: There were decreases in chymotrypsin-like activity of $20 \mathrm{~S}$ rabbit muscle proteasomes with thiostrepton, rifampicin, 2-hydroxyestradiol, 2-methoxyestradiol, 25-hydroxycholesterol, nicotinic acid, vitamin $\mathrm{D}_{3}$, trans-resveratrol $(35 \%-68 \% ; \mathrm{P}<0.001)$, and increases with (-) corey lactone, ouabain, ampicillin, ascorbic acid, codeine, amiloride-HCL $(121 \%-275 \%$; P < 0.001$)$ in $20 \mathrm{~S}$ proteasomes. All these compounds inhibited TNF- $\alpha$ secretion $(33 \%-75 \% ; \mathrm{P}<0.001)$ in lipopolysaccharide-induced macrophages of C57BL/6 mice (Wild Type). However, these compounds activated (127\% - 190\% P < 0.01), or inhibited secretion of TNF- $\alpha(48 \%-78 \%$; $\mathrm{P}<0.001)$, and production of nitric oxide $(37 \%-77 \% ; \mathrm{P}<0.001)$ in lipopolysaccharide-induced macrophages from PPAR- $\alpha$ knockout mice. Gene expression results of TNF- $\alpha$, IL- $1 \beta$, IL- 6 , and iNOS activity were consistent with results obtained for TNF- $\alpha$ protein and NO production as observed with macrophages of PPAR- $\alpha$ knockout mice. The possible mechanism for inhibition is due to decreased proteolytic degradation of $\mathrm{p}$-I $\mathrm{KB}$ protein, followed by decreased translocation of activated NF- $\kappa \mathrm{B}$, and depressed transcription of gene expression of TNF- $a$, and iNOS activity.

Conclusions: The present results have provided two sets of compounds, anti-inflammatory (for the control of diabetes and cardiovascular disease), and pro-inflammatory for the treatment of other diseases.

Keywords: Selected compounds; TNF- $\alpha$; nitric oxide; IL-1 $\beta$, IL-6; iNOS

List of Abbreviations: LPS: lipopolysaccharide; TNF- $\alpha$ : tumor necrosis factor- $\alpha$; IL-1 $\beta$ : interleukin-1 $\beta$; IL-6: interleukin-6; NO: nitric oxide; iNOS: inducible nitric oxide synthase; NF- $\kappa$ B: nuclear factor-kappaB; p-IкB: phosphorylated-inhibitor kappaB; PPAR- $\alpha$ knockout mice: peroxisome proliferator-activated receptor-a knockout mice; TG: thioglycolate
\end{abstract}

\section{Introduction}

We have recently described that naturally-occurring compounds play an important therapeutic role in modulating the inflammatory biomarkers in cardiovascular disease, diabetes and cancer [1]. Those compounds were able to inhibit or activate secretion of TNF- $\alpha$, and inhibit production of nitric oxide (NO) in murine cell line (RAW264.7), and in lipopolysaccharide-induced thioglycolate (TG)-elicited peritoneal macrophages prepared from C57BL/6 (wild type; control group), BALB/c, double subunits knockout (LMP-7/MECL-1 ${ }^{-/}$) mice [1]. However, in TG-elicited peritoneal macrophages obtained from peroxisome proliferator activated receptor- $\alpha$ (PPAR- $\alpha$ ) knockout female mice, the secretion of TNF- $\alpha$ was activated by some of the compounds rather than inhibited, as compared to control (C57BL/6), and other groups [1]. The important role played by lipopolysaccharides (LPS) in up-regulating inflammation is well-established [2]. In short, LPS is expressed on the outer membrane of gram-negative bacteria. LPS induces several pro-inflammatory cytokines, such as tumor necrosis factor- $\alpha$ (TNF- $\alpha$ ), interleukin-1 $\beta$ (IL-1 $\beta$ ), IL-6, IL- 8 and production of nitric oxide [2]. 
In order to find potent inflammatory biomarkers, we have selected 32 compounds of different categories of organic chemistry as shown in Table 1. The PPAR- $\alpha$ knockout female mice were selected for present study due to their different effects in LPSinduced macrophages of $\delta$-tocotrienol, riboflavin, quercetin on secretion of TNF- $\alpha$ activation compared to corresponding wild type (C57BL/6) control (inhibition) group, and also due to the prolonged response to inflammatory stimuli [3]. Moreover, the PPARs mice contain nuclear receptors, which bind to fatty acid-derived ligands and activate the transcription of genes that govern lipid metabolism. The primary sites of activation of PPAR- $\alpha$, which recognizes monounsaturated and polyunsaturated fatty acids and eicosanoids, are present in liver, heart, muscle, and kidney.

According to its role in regulating fatty acid metabolism, PPAR- $\alpha$ activates gene expression involved in fatty acid uptake (fatty acid binding protein), $\beta$-oxidation (medium chain acyl-CoA dehydrogenase, carnitine palmitoyl transferase I, and acyl-CoA oxidase), transport into peroxisomes (ATP-binding cassette transporters $\mathrm{D}_{2}$ and $\mathrm{D}_{3}$ ), and omega-oxidation of unsaturated fatty acids (cytochrome P-450, 4A1 and 4A3). Moreover, PPAR- $\alpha$ also induce fatty acid catabolism and prevent hypertriglyceridemia, and its activation decreases glucose uptake, and causes a shift in glucose use to fatty acid oxidation in cardiac muscle. Therefore, selective PPAR- $\alpha$ agonists that increase fatty acid catabolism without using lipid accumulation in the heart might be effective treatment for dyslipidemia [3].

The objective of present study was to evaluate the effects of 22 compounds (Table 1A) on the inhibition/activation of proteasome activities, secretion of TNF- $\alpha$ production of nitric oxide (NO), certain anti-inflammatory/pro-inflammatory cytokines (IL-1 $\beta$, IL-6) and iNOS enzyme activity by using TG-elicited peritoneal macrophages prepared from PPAR- $\alpha$ knockout female mice. It has been reported that proteasome is a hollow, complex, regulatory protein consisting of three proteolytic subunits, $\mathrm{X}$, $\mathrm{Y}$, Z, with chymotrypsin-like, trypsin-like, and post-glutamase activities, respectively. Lactacystin is a potent known inhibitor of chymotrypsin-like activity of $20 \mathrm{~S}$ proteasome and therefore we included lactacystin as a positive inhibitor in the current study [4]. Lactacystin (lactone)as a proteasome inhibitor also known to be responsible for secretion of TNF- $\alpha$, and nitric oxide production [5]. As described earlier, that nitric oxide production increases during ageing process, which could be due to a diminished activation of NF- $\kappa B$ signaling [6-8]. Therefore, it was suggested that above mentioned compounds may also block the activation of NF- $\kappa \mathrm{B}$, thus resulting lowering of serum TNF- $\alpha$ and nitric oxide (NO) levels in experimental models. The important role of NF- $\kappa B$ in various biological functions has been reported [9]. The data on the effect of these modulators in secretion of TNF- $\alpha$, and inhibition of nitric oxide production by these compounds may be of clinical relevance in host defense mechanisms against various infections, and therapy for several inflammatory diseases $[10,11]$.

\begin{tabular}{|c|c|}
\hline \# & Section I: Known Proteasome Inhibitors \\
\hline 1 & Lactacystin \\
\hline 2 & Dexamethasone \\
\hline & Section II: Known Proteasome stimulators \\
\hline 3 & (-) Corey Lactone \\
\hline 4 & Ouabain \\
\hline & Section III: Antibiotics \\
\hline 5 & Thiostrepton \\
\hline 6 & Rifampicin \\
\hline 7 & Ampicillin \\
\hline & Section IV: Cholesterol Inhibitors \\
\hline 8 & Mevinolin (Lovastatin) \\
\hline 9 & 2-Hydroxyestradiol \\
\hline 10 & 2-Methoxyestradiol \\
\hline 11 & 25-Hydroxycholesterol \\
\hline & Section V: Antioxidants \\
\hline 12 & Acetylsalicylic Acid (Aspirin) \\
\hline
\end{tabular}

\begin{tabular}{|c|c|}
\hline \# & Section I: Known Proteasome Inhibitors \\
\hline 1 & Lactacystin \\
\hline & Section II: Known Proteasome stimulators \\
\hline 2 & (-) Corey Lactone \\
\hline 3 & Ouabain \\
\hline & Section III: Antibiotics \\
\hline 4 & Thiostrepton \\
\hline 5 & Rifampicin \\
\hline 6 & Ampicillin \\
\hline & Section IV: Cholesterol Inhibitors \\
\hline 7 & 2-Hydroxyestradiol \\
\hline 8 & 2-Methoxyestradiol \\
\hline 9 & 25-Hydroxycholesterol \\
\hline
\end{tabular}




\begin{tabular}{|c|c|}
\hline 13 & a-Tocopherol \\
\hline 14 & $\gamma$-Tocotrienol \\
\hline \multirow[t]{3}{*}{15} & $\delta$-Tocotrienol \\
\hline & Section VI: Vitamins \\
\hline & Water Solubles \\
\hline 16 & Ascorbic Acid (Vitamin C) \\
\hline 17 & Riboflavin (Vitamin $\mathrm{B}_{2}$ ) \\
\hline \multirow[t]{3}{*}{18} & Niacin (Vitamin $B_{3}$ ) \\
\hline & Section VII: Vitamins \\
\hline & Water Insolubles \\
\hline 19 & Vitamin $\mathrm{D}_{3}$ \\
\hline \multirow[t]{4}{*}{20} & Vitamin E \\
\hline & $\alpha$-. $\beta$ -.$\gamma$-. $\delta$-Tocopherols \\
\hline & $\alpha-\beta$ - $\beta$ -.$\delta$-Tocotrienols \\
\hline & Section VIII: Polyphenols \\
\hline 21 & Quercetin Sulphate \\
\hline 22 & trans -Resveratrol \\
\hline 23 & trans -Petrostilbene \\
\hline \multirow[t]{2}{*}{24} & Morin Hydrate \\
\hline & Section IX: Alkaloids + Narcotics \\
\hline 25 & Vincaleucoblastin Sulphate \\
\hline \multirow[t]{2}{*}{26} & Codeine \\
\hline & Section X: Neurotransmiter \\
\hline \multirow[t]{2}{*}{27} & Dopamine-HCL \\
\hline & $\begin{array}{l}\text { Section XI: Miscellaneous Useful } \\
\text { Pharmaceutical Products }\end{array}$ \\
\hline 28 & Quinine Sulphate \\
\hline 29 & Amiloride \\
\hline 30 & $\alpha$-Lipoic Acid \\
\hline 31 & Coenzyme Q10 \\
\hline 32 & Hydrochlorothiazide-HCL \\
\hline
\end{tabular}

Table 1: Evaluation of following compounds on several inflammatory biomarkers in PPAR- $\alpha$ a knockout mice

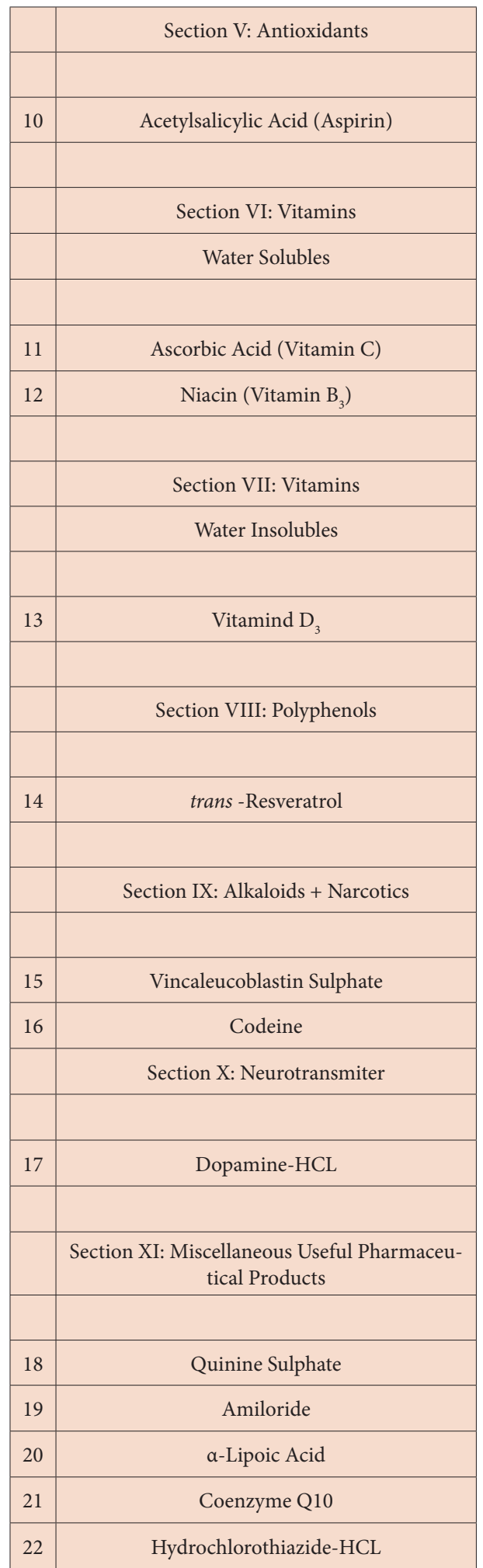

Table 1A: Evaluation of following compounds on severa inflammatory biomarkers in PPAR- $\alpha$ knockout mice

\section{Materials and Methods}

\section{Reagents}

The deep rough chemotype LPS (Re LPS) from E. coli D31M4 was purified as reported earlier [4]. Dulbecco's Modified Eagle Medium (DMEM) heat-inactivated low-endotoxin fetal bovine serum (FBS), and gentamicin were purchased from Cambrex (Walkersville, MD, USA) for tissue culture studies. Thioglycolate (TG) was purchased from Sigma, Aldrich Chemical Co. (St. Louis, MO, USA) and RNeasy mini kit from QIAGEN Sciences (Germantown, MD, USA). Most of the compounds were purchased from Sigma- 
Aldrich Chemical Co (St. Louis, MO, USA). Codeine and dopamine-HCL were obtained from the Department of Pathology, School of Medicine, Kansas City, MO after completing all the required formalities to use this compound in the laboratory.

\section{Animals}

The PPAR- $\alpha$ knockout female mice were selected for the present study due to their differential effects with $\delta$-tocotrienol, riboflavin, quercetin on the secretion of TNF- $\alpha$ compared to corresponding wild type (C57BL/6) control group in LPS-induced macrophages [1]. The 6-week-old C57BL/6 female mice (Wild Type; control group) were purchased from the Jackson laboratory (Bar Harbor, ME, USA), and peroxisome proliferator-activated receptor- $\alpha$ (PPAR- $\alpha$ ) knockout female mice were bred at UMKC's Animal Facility (Kansas City, MO, USA). Mice used in this study received humane care in compliance with the principles of laboratory animal care formulated by the National Society of Health Guide for the "Care and Use of Laboratory Animals" by the US National Society of Health (NIH Publication No 85-23, revised 1996). The experimental procedures involving animals were reviewed and approved by the "Institutional Animal Care and Use Committee of UMKC", Medical School, MO. USA.

All 6-week-old C57BL/6 $(\mathrm{n}=8)$, and PPAR- $\alpha$ knockout female mice $(\mathrm{n}=20)$ were acclimatized to new environment for 14 days before beginning experimentation. The mice were fed ad libitum regular commercial mouse diet and had free access to water throughout the experiment. A $12 \mathrm{~h}$ light and $12 \mathrm{~h}$ dark cycle was maintained during feeding period.

\section{Effects of selected compounds on chymotrypsin-like activity of 20S rabbit muscle proteasome}

First, a comparative effectiveness of different doses $(20 \mu \mathrm{M}-320 \mu \mathrm{M})$ of following compounds; lactacystin, (-) corey lactone, ouabain, thiostrepton, rifampicin, ampicillin, 2-hydroxyestradiol, 2-methoxyestradiol, 25-hydroxycholesterol, acetylsalicylic acid, ascorbic acid, nicotinic acid, vitamin $\mathrm{D}_{3}$, trans-resveratrol on the suppression of chymotrypsin-like activity of $20 \mathrm{~S}$ rabbit muscle proteasome was evaluated. For these studies, different concentrations of each compound were prepared in media containing $0.5 \%$ DMSO. The proteasomal activity of the $20 \mathrm{~S}$ rabbit muscle proteasome $(0.4 \mu \mathrm{g} / \mathrm{ml})$ were assayed with synthetic peptide substrate in $0.02 \mathrm{M}$ Tris-HCl buffer ( $\mathrm{pH}$ 7.2). The substrate used for the chymotrypsin-like activity was $100 \mu \mathrm{M}$ of succinyl-Leu-Leu-Val-Tyramino methyl coumarin. Fluorescence was measured (absorption at $360 \mathrm{~nm}$ and emission at $460 \mathrm{~nm}$ ) using an Flx $800 \mathrm{microplate}$ fluorescence reader (Bio-Tek Instruments, Winooski, VT, USA).

\section{Methodology for use of selected compounds on secretion of TNF- $\alpha$ in LPS-induced TG-elicited peritoneal macrophages of 8-week-old female C57BL/6, and PPAR- $\alpha$ knockout mice}

The TG-elicited peritoneal macrophages were adhered to bottom of $100 \mathrm{~mm}$ tissue culture plates $\left(1 \mathrm{x} 10^{7}\right.$ cells/well in $1.0 \mathrm{ml}$ medium) for $4 \mathrm{~h}$, the supernatants were removed, and cells were washed extensively with medium three times. The cells were cultured overnight in fresh media after final wash. After overnight incubation at $37^{\circ} \mathrm{C}$ in presence of $\mathrm{CO}_{2}$, cells were treated with various concentrations of each compound $(100 \mu \mathrm{l}$ of $20 \mu \mathrm{M}$ to $320 \mu \mathrm{M}$ dissolved in $0.2 \%$ DMSO) and LPS (10.0 $\mu \mathrm{l} \mathrm{of} 1.0 \mu \mathrm{g} / \mathrm{ml}$ of stock solution; $10.0 \mathrm{ng} /$ well) of each compound. The supernatants were collected after $4 \mathrm{~h}$ of incubation at $37^{\circ} \mathrm{C}$ in presence of $\mathrm{CO}_{2}$, and after $4 \mathrm{~h}$, the levels of TNF- $\alpha$ in supernatants were measured by Quantikine M ELISA kit (R\&D System, Minneapolis, MN, USA) according to manufacturer's instructions. The lower limit of detection for TNF- $\alpha$ in this method is $5.0 \mathrm{pg} / \mathrm{ml}$ [12]. The viability of peritoneal macrophages treated with various compounds plus LPS were also determined by trypan blue dye exclusion or a quantitative colorimetric assay with 3-(4,5)-dimethylthiozol-2,5-diphenyl-tetrazolium bromide (MTT) as described previously [5].

\section{Methodology for effect of selected compounds on production of nitric oxide in LPS-induced TG-elicited peritoneal macrophages of 8-week-old female PPAR- $\alpha$ knockout mice}

Similarly, TG-elicited peritoneal macrophages ( 1 x $10^{7}$ cells/well in $500 \mu$ medium [DMEM]) were adhered to bottom of $100 \mathrm{~mm}$ tissue culture plates for $2 \mathrm{~h}$. After $2 \mathrm{~h}$, the cells were treated with $100 \mu \mathrm{l}$ of various concentrations of each compound (dissolved in $0.2 \% \mathrm{DMSO}$ ) plus LPS (10.0 ng/well in $400 \mu \mathrm{l})$. The assay mixtures were incubated at $37^{\circ} \mathrm{C}$ in presence of $\mathrm{CO}_{2}$ for $36 \mathrm{~h}$. After $36 \mathrm{~h}$, the levels of nitric oxide (NO) were determined by measuring the amount of nitrite, a stable metabolic product of nitric oxide, as previously reported [10]. The assay mixture contained medium $(100 \mu \mathrm{l})$ plus Griess reagent $(100 \mu \mathrm{l})$, and absorption was measured at $570 \mathrm{~nm}$ using a "Microplate Reader" (MR 5000; Dynatech Labs, Inc. USA). The amount of nitrite was determined by comparison of unknowns using a $\mathrm{NaNO}_{2}$ standard curve. The NO detection limit was $0.20 \mathrm{nM}$ [11].

\section{Methodology for effect of selected compounds on time-dependent secretion of TNF- $\alpha$ in LPS-induced TG-elicited peritoneal macrophages of 8-week-old female PPAR- $\alpha$ knockout mice}

The TG-elicited peritoneal macrophages prepared from 8-week-old female PPAR- $\alpha$ knockout mice were adhered to the bottom of $100 \mathrm{~mm}$ tissue culture plates $\left(1 \times 10^{7} /\right.$ well in $1.0 \mathrm{ml}$ medium) for $4 \mathrm{~h}$. After four $\mathrm{h}$, supernatants were removed, and cells were washed with medium three times. Cells were cultured overnight in fresh medium after the final wash. After overnight incubation at $37^{\circ} \mathrm{C}$ in presence of $\mathrm{CO}_{2}$, the cells were treated with various compounds (dissolved in $0.2 \%$ DMSO) and LPS (10.0 ng/well). The supernatants were collected after $1 \mathrm{~h}, 2 \mathrm{~h}$, and $3 \mathrm{~h}$ incubation at $37^{\circ} \mathrm{C}$ in presence of $\mathrm{CO}_{2}$ to carry out TNF- $\alpha$ estimation by using ELISA assay kit. The cells viabilities were also determined by MTT [12]. 


\section{Methodology for effects of selected compounds on gene expression of TNF- $\alpha$, IL- $1 \beta$, IL- 6 and iNOS in LPS-induced TG-elicited peritoneal macrophages of 8-week-old female PPAR- $\alpha$ knockout mice}

The various concentrations of each compound were dissolved in medium containing 0.2\% DMSO. TG-elicited peritoneal macrophages were prepared from 8-week-old female PPAR- $a$ knockout mice as described previously [1,4]. The macrophages (1 $\mathrm{x} 10^{6}$ cells/well in $500 \mu \mathrm{l}$ medium) were adhered in wells with various concentrations of each compound for $2 \mathrm{~h}$. Then all wells were challenged with LPS $(10.0 \mathrm{ng} /$ well; $400 \mu \mathrm{L})$, and incubated at room temperature for $4 \mathrm{~h}$. After $4 \mathrm{~h}$, assay mixtures were centrifuged at 2,000 rpm for $20 \mathrm{~min}$. Cells were harvested, and total cellular RNA was extracted from each pellet with RNeasy mini kit (QIAGEN Sciences; Germantown, MD, USA) according to instructions of manufacturer. The RNA of each treatment was transcribed and resulting data was amplified and analyzed by real-time polymerase chain reaction (RT-PCR) to quantitate gene expression of TNF- $\alpha$, IL-1 $\beta$, IL-6 and iNOS by using 1-step RT-PCR kit (QIAGEN, Chatsworth, CA, USA) according to the manufacturer's instructions $[4,13,14]$.

\section{Detection of cell viability}

The viability of TG-elicited peritoneal macrophages derived from PPAR- $\alpha$ knockout female mice treated with LPS plus various compounds was determined by trypan blue dye exclusion or a quantitative colorimetric assay with 3-(4,5)-dimethyl-thiozol-2, 5diphenyltetrazolium bromide MTT [4,12].

\section{Statistical Analyses}

Stat View software (version 4.01, Abacus Concepts, Berkeley, CA) was used for the analyses of treatment-mediated effects as compared to control group. Treatment-mediated differences were detected with a one-way ANOVA, and when the F test indicated a significant effect, differences between means were analyzed by a Fisher's protected least significant difference test. Data were reported as means \pm SD in text and Tables. The statistical significant level was set at $5 \%(\mathrm{P}<0.05)$.

\section{Results}

\section{Effect of selected compounds on chymotrypsin-like activity of 20S rabbit muscle proteasome}

As mentioned earlier, lactacystin was included in this study, because it is a known selective inhibitor of chymotrypsin-like activity of proteasome and was used as a positive control. We first screened several compounds for chymotrypsin-like activity of $20 \mathrm{~S}$ rabbit muscle proteasome. The $20 \mathrm{~S}$ proteasome activity was measured after treatment with these compounds at concentrations ranging from $2.5 \mu \mathrm{M}$ to $160 \mu \mathrm{M}$ compared to activity of control. Results of this study revealed dose-dependent decreases of chymotrypsin-like activity of $20 \mathrm{~S}$ rabbit muscle proteasomes between $2.5 \mu \mathrm{M}$ and $40 \mu \mathrm{M}$ for most of the compounds, except for acetylsalicylic acid (aspirin, $160 \mu \mathrm{M}$ ) compared to respective controls (Table 2). The most effective decrease in chymotrypsinlike activity of $20 \mathrm{~S}$ proteasome was observed with thiostrepton $(5 \mu \mathrm{M}>50 \%)$, rifampicin $(20 \mu \mathrm{M})$, 25-hydroxycholesterol $(20$ $\mu \mathrm{M}$ ), and trans-resveratrol compared to respective controls (Table 2). The effects of (-) corey lactone, ouabain, ampicillin (broadspectrum antibiotic), ascorbic acid (vitamin C), codeine, and amiloride-HCL showed increases between $(10 \mu \mathrm{M}-40 \mu \mathrm{M})$ in chymotrypsin-like activity of $20 \mathrm{~S}$ proteasome (Table 2 ). These results indicate the capacity of these compounds to inhibit or activate 20S chymotrypsin-like activity at various concentrations. Therefore, in subsequent studies, most effective single dose of these compounds (14 out of 22) was selected to evaluate effects on inflammatory biomarkers, as shown in Table 3 and Figure 1. The remaining eight compounds were not tested in subsequent experimentation because either these were less effective or were not commercially available (vincaleucoblastin, codeine, and dopamine-HCL). The most impressive significant $(\mathrm{P}<0.01-0.001)$ decrease in $20 \mathrm{~S}$ proteasome activity were observed using various concentrations of each compound, such as with thiostrepton (35\%), rifampicin (44\%), 2-hydroxyestradiol (47\%), 2-methoxyestradiol (40\%), 25-hydroxycholesterol (39\%), nicotinic acid (51\%), vitamin $\mathrm{D}_{3}(68 \%)$ and trans-resveratrol (59\%) compared to respective controls (Table 3 and Figure 1). Similarly, the most significant increases in $20 \mathrm{~S}$ activity were obtained with $(-)$ corey lactone $(138 \%$; $\mathrm{P}<0.01)$, ouabain $(153 \% ; \mathrm{P}<0.001)$, ampicillin $(149 \%$; $\mathrm{P}<$ $0.001)$, and ascorbic acid $(168 \%$; $\mathrm{P}<0.001)$ compared to controls (Table 3, Figure 1). Similar down-regulation or upregulation was observed in the secretion of TNF- $\alpha$ by these compounds, but all compounds inhibited induction of nitric oxide and iNOS enzyme activity in LPS-induced TG-elicited peritoneal macrophages from 8-week-old female PPAR- $\alpha$ knockout mice, as described below.

\section{Dose-dependent evaluation of selected compounds on secretion of TNF- $\alpha$ in LPS-induced TG-elicited peritoneal macrophages obtained from 8-week-old female PPAR- $\alpha$ knockout mice}

We next determined effects of these 14 compounds (Table 3) on secretion of TNF- $\alpha$ using concentrations between $2.5 \mu \mathrm{M}-160 \mu \mathrm{M}$ for all the compounds except acetylsalicylic acid $(10 \mu \mathrm{M}-640 \mu \mathrm{M})$ in LPS-induced TG-elicited peritoneal macrophages obtained from 8-week-old female PPAR- $\alpha$ knockout mice. There were dose-dependent increases in the secretion of TNF- $\alpha$ by (-) corey lactone, ouabain, ampicillin and ascorbic acid, and decreases by rest of compounds compared to respective controls (Table 4), which were similar to $20 \mathrm{~S}$ activity (Table 3 ) as observed earlier. The most effective doses for the increases or decreases were between $20 \mu \mathrm{M}-40$ $\mu \mathrm{M}$ for all the compounds, except acetylsalicylic acid (aspirin) showed maximum decrease with $320 \mu \mathrm{M}$ (Table 4). The results of these compounds are similar as reported for dexamethasone, mevinolin, $\delta$-tocotrienol, riboflavin and quercetin-HCL in LPS-induced peritoneal macrophages of PPAR-a knockout mice, which are different as compared to its control C57BL/6 (Wild Type) mice [1]. 


\begin{tabular}{|c|c|c|c|c|c|c|c|c|c|c|c|}
\hline$\#$ & & 1 & 2 & 3 & 4 & 5 & 6 & 7 & 8 & 9 & 10 \\
\hline & Assay Mixture & $\begin{array}{l}\text { 20S Protea- } \\
\text { some }(20 \\
\text { SP })^{1}\end{array}$ & $\begin{array}{c}\text { Tris + 20SP } \\
\quad=\mathrm{A}^{2}\end{array}$ & $\begin{array}{c}\mathrm{A}+0.5 \% \mathrm{DMSO}^{3} \\
=\mathrm{B}\end{array}$ & $\mathrm{B}+2.5 \mu \mathrm{M}$ & $\mathrm{B}+5 \mu \mathrm{M}$ & $\mathrm{B}+10 \mu \mathrm{M}$ & $\mathrm{B}+20 \mu \mathrm{M}$ & $\mathrm{B}+40 \mu \mathrm{M}$ & $\mathrm{B}+80 \mu \mathrm{M}$ & $B+160 \mu M$ \\
\hline 1 & Lactacystin & 12610 & 12106 & $13075(100)^{4}$ & $9298(71)$ & $4779(37)$ & $4214(32)$ & $3950(30)$ & $3390(26)$ & 3025 (23) & $2578(20)$ \\
\hline 2 & (-) Corey Lactone & 18486 & 15259 & $17792(100)$ & $18860(106)$ & 20461 (115) & $22240(125)$ & $24553(138)$ & $28645(161)$ & $25087(141)$ & 32915 (185) \\
\hline 3 & Ouabain & 18321 & 15365 & $16193(100)$ & 20565 (127) & 21375 (132) & $23642(146)$ & 24775 (153) & $28176(174)$ & $30281(187))$ & 32224 (199) \\
\hline 4 & Thiostrepton & 20291 & 19563 & $17752(100)$ & $9233(52)$ & $6274(35)$ & $3193(18)$ & $1978(11)$ & $1642(9)$ & $1487(8)$ & $1197(7)$ \\
\hline 5 & Rifampicin & 24472 & 13548 & $15633(100)$ & $13006(83)$ & $10196(65)$ & $9861(63)$ & $6861(44)$ & $4680(30)$ & $864(6)$ & $644(4)$ \\
\hline 6 & Ampicillin & 18727 & 17526 & $17526(100)$ & $21840(125)$ & $22224(127)$ & $22716(130)$ & $25068(143)$ & 26068 (149) & $29294(167)$ & 23869 (136) \\
\hline 7 & 2-hydroxyestradiol & 22456 & 13567 & $16873(100)$ & $14754(87)$ & $13890(82)$ & $11589(69)$ & $9823(58)$ & $7895(47)$ & $6937(41)$ & $5691(34)$ \\
\hline 8 & 2-hydroxyestradiol & 25891 & 14957 & $18246(100)$ & 18057 (99) & $17236(94)$ & $16429(90)$ & $14822(81)$ & $13976(77)$ & $7331(40)$ & $3746(21)$ \\
\hline 9 & $\begin{array}{l}\text { 25-Hydroxycho- } \\
\text { lesterol }\end{array}$ & 23801 & 22524 & $19352(100)$ & $16515(85)$ & $13360(69)$ & $11000(57)$ & $7486(39)$ & 7457 (39) & $5689(29)$ & $4876(25)$ \\
\hline 10 & Acetylsalicylic Acid & 24021 & 17197 & $13804(100)$ & $12700(92)$ & $12424(90)$ & $11871(86)$ & $10077(73)$ & $9801(71)$ & $9249(67)$ & $8835(64)$ \\
\hline 11 & $\begin{array}{l}\text { Ascarbic Acid } \\
\text { (vitamin C) }\end{array}$ & 18992 & 16326 & $11812(100)$ & $16859(143)$ & $17492(145)$ & $19887(168)$ & $19185(162)$ & $15751(133)$ & $14493(123)$ & $13593(115)$ \\
\hline 12 & $\begin{array}{l}\text { Nicotinic Acid } \\
\text { (vitamin B2) }\end{array}$ & 19618 & 19508 & $14412(100)$ & $9729(68)$ & $8975(62)$ & $8341(58)$ & $7375(51)$ & $11994(83)$ & $14017(97)$ & $15218(106)$ \\
\hline 13 & Vitamin $\mathrm{D}_{3}$ & 19732 & 15981 & $16378(100)$ & $15887(97)$ & $14085(86)$ & $13430(82)$ & $11623(71)$ & $11137(68)$ & $11465(70)$ & $4429(52)$ \\
\hline 14 & trans-Resveratrol & 18486 & 15433 & $17792(100)$ & $14412(81)$ & $14234(80)$ & $11921(67)$ & $10497(59)$ & $9786(55)$ & $8086(45)$ & $7295(41)$ \\
\hline 15 & Vincaleucoblastin & 20874 & 20246 & $15826(100)$ & $14560(92)$ & $13927(88)$ & $12819(81)$ & $11711(74)$ & $10445(66)$ & $10129(64)$ & $9021(57)$ \\
\hline 16 & Codeine & 19444 & 17784 & $16793(100)$ & 16625 (99) & $18472(110)$ & $18976(113)$ & $20152(120)$ & $20320(121)$ & 20488 (122) & $20823(124)$ \\
\hline 17 & Dopamine-HCL & 21486 & 20648 & $17792(100)$ & $15657(88)$ & $14412(81)$ & $12277(69)$ & $10675(60)$ & $9964(56)$ & $8086(45)$ & $6227(35)$ \\
\hline 18 & Quinine Sulphate & 23586 & 22465 & $21876(100)$ & 20345 (93) & $18761(86)$ & $18813(83)$ & $17938(82)$ & $17720(81)$ & $17063(78)$ & $16188(74)$ \\
\hline 19 & Amiloride-HCL & 20486 & 21259 & $18122(100)$ & $20478(113)$ & $21384(118)$ & $35700(197)$ & $49836(275)$ & $74300(410)$ & $114712(633)$ & $123230(680)$ \\
\hline 20 & $\alpha$-Lipoic Acid & 22762 & 21982 & $20542(100)$ & $18932(92)$ & $17461(85)$ & $17255(84)$ & $16639(81)$ & $16228(79)$ & $16023(78)$ & $16228(79)$ \\
\hline 21 & coenzyme Q10 & 20872 & 20871 & $18233(100)$ & $17139(94)$ & $15680(86)$ & $15498(85)$ & $15315(84)$ & $14951(82)$ & $14769(81)$ & $14586(80)$ \\
\hline 22 & $\begin{array}{l}\text { Hydrochlorothi- } \\
\text { azide-HCL }\end{array}$ & 24871 & 20259 & $17792(100)$ & $14945(84)$ & $16191(91)$ & $15301(86)$ & $12099(68)$ & $11743(66)$ & $11209(63)$ & $10675(60)$ \\
\hline
\end{tabular}

${ }^{1}$ Chymotrypsin-like activity was carried out by using synthetic dipeptide substrate III (Suc-Leu-Leu-Val-Tyr-AMC) in Tris buffer $\mathrm{pH}$

7.5, $0.02 \mathrm{M}$. Rabbit muscle proteasome was used. Time of incubation was 30 minutes

${ }^{2}$ Fluorescence absorption was measured at excitation $=360 \mathrm{~nm}$ and emission $=460 \mathrm{~nm}$

${ }^{3}$ Each compound was dissolved in dimethyl sulfuoxide (0.5\% DMSO)

${ }^{4}$ Percentages of control values are in parentheses

Table 2: Effects of selected compounds on the chymotrypsin-like activity of 20S rabbit muscle proteasome

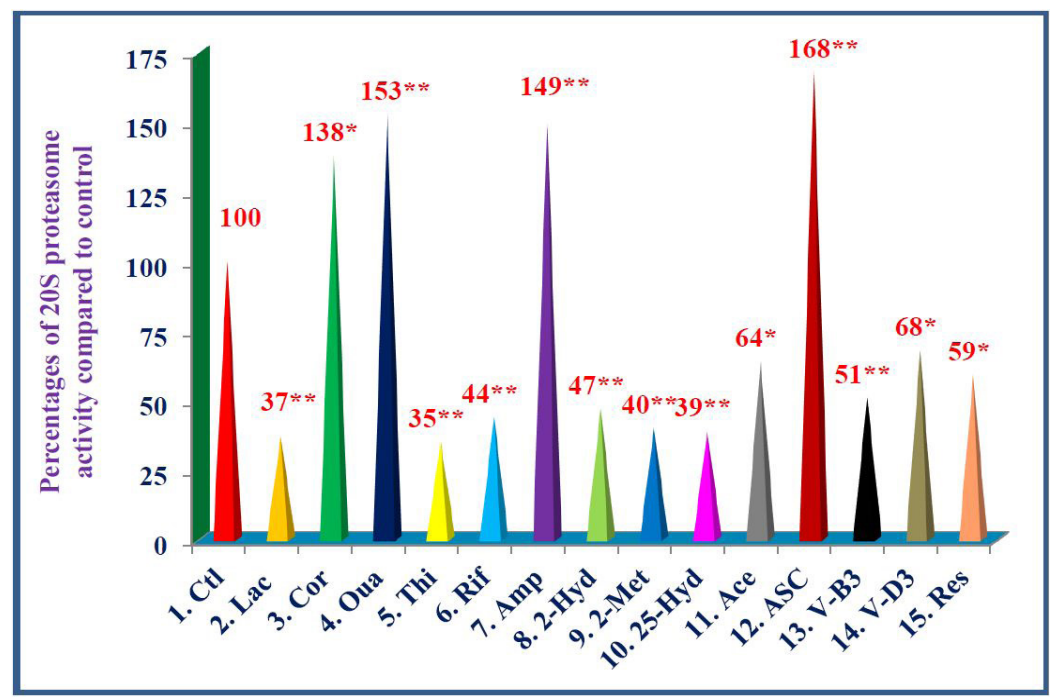

Figure 1: Effects of selected compounds on chymotrypsin-like activity of 20S rabbit muscle proteasome

The $20 \mathrm{~S}$ rabbit muscle proteasome was treated with various compounds $(100 \mu \mathrm{l})$ dissolved in $0.5 \% \mathrm{DMSO}$ of 3 . lactacystin $(5 \mu \mathrm{M})$; 4 . (-) corey lactone $(20 \mu \mathrm{M})$; 5. ouabain $(20 \mu \mathrm{M}) ; 6$. thiostrepton $(5 \mu \mathrm{M})$; 7. rifampicin $(20 \mu \mathrm{M}) ; 8$. ampicillin $(40 \mu \mathrm{M}) ; 9$. 2-hydroxyestradiol $(40 \mu \mathrm{M}) ; 10$. 2 -methoxyestradiol $(80 \mu \mathrm{M}) ; 11$. 25-hydroxycholesterol $(20 \mu \mathrm{M}) ; 12$. acetylsalicylic acid $(160 \mu \mathrm{M}) ; 13$. ascorbic acid $(10 \mu \mathrm{M}) ; 14$. nicotinic acid $(20 \mu \mathrm{M}) ; 15$. vitamin $\mathrm{D}_{3}(40 \mu \mathrm{M}) ; 16$. trans-resveratrol $(20 \mu \mathrm{M})$ for $30 \mathrm{~min}$. The proteolytic activity was measured by adding succinyl-Leu-Leul-Val-Tyr-amino methyl coumarin as substrate and fluorescence (absorption at $360 \mathrm{~nm}$ and emission at $460 \mathrm{~nm}$ ) was measured by using Flx 800 microplate fluorescence reader. Data are average of triplicate analyses of each sample as \pm SD (standard deviation). Percentage values of each treatment compared to control are at the top of the column. Values in a column not sharing a common asterisk are significantly different at ${ }^{*} \mathrm{P}<0.01 ;{ }^{*} \mathrm{P}<0.001$. 


\begin{tabular}{|c|c|c|c|c|c|}
\hline & Assay Mixture & $\mathbf{\mu M}$ & $\mathbf{1 0 0 \%}$ & $\mathbf{2 0 S}_{\text {proteasome }}{ }^{2}$ & Percentages \\
\hline 1 & Tris + 20S proteasome $=\mathrm{A}$ & & 0 & 0.00 & 0.00 \\
\hline 2 & A + 0.5\% DMSO ${ }^{3}=\mathrm{B}$ & & $(15989 \pm 2254)$ & & 100 \\
\hline 3 & B + Lactacystin & 5 & 13075 & $4779 \pm 456^{* *}$ & 37 \\
\hline 4 & B + (-) Corey Lactone & 20 & 17792 & $24553 \pm 2792^{*}$ & 138 \\
\hline 5 & B + Ouabain & 20 & 16293 & $24928 \pm 2955^{* *}$ & 153 \\
\hline 6 & B + Thiostrepton & 5 & 17752 & $6274 \pm 886^{* *}$ & 35 \\
\hline 7 & B + Rifampicin & 20 & 15633 & $6861 \pm 784^{* *}$ & 44 \\
\hline 8 & B + Ampicillin & 40 & 17526 & $26068 \pm 3256^{* *}$ & 149 \\
\hline 9 & B + 2-Hydroxyestradiol & 40 & 16873 & $7895 \pm 859^{* *}$ & 47 \\
\hline 10 & B + 2-Methoxyestradiol & 80 & 18246 & $7331 \pm 776^{* *}$ & 40 \\
\hline 11 & B + 25-Hydroxycholestrol & 20 & 19352 & $7486 \pm 793^{*}$ & 39 \\
\hline 12 & B + Acetylsalicylic Acid & 160 & 13804 & $8835 \pm 854^{*}$ & 64 \\
\hline 13 & B + Ascorbic Acid (Vitamin C) & 10 & 11812 & $19887 \pm 2067^{* *}$ & 168 \\
\hline 14 & B + Nicotinic Acid (Vitamin $\mathrm{B}_{3}$ ) & 20 & 14421 & $7375 \pm 849^{* *}$ & 51 \\
\hline 15 & B + Vitamin $\mathrm{D}_{3}$ & 40 & 16378 & $11137 \pm 962^{*}$ & 68 \\
\hline 16 & B + trans -Resveratrol & 20 & 17793 & $14828 \pm 1174^{*}$ & 59 \\
\hline
\end{tabular}

${ }^{1}$ Chymotrypsin-like activity was carried out by using synthetic dipeptide sustrate III (Suc-Leu-Leu-Val-Tyr-AMC) in Tris buffer pH 7.5, $0.02 \mathrm{M}$. Rabbit muscle proteasome was used. Time of incubation was 30 minutes

${ }^{2}$ Fluorescence absorption was measured at excitation $=360 \mathrm{~nm}$ and emission $=460 \mathrm{~nm}$

${ }^{3}$ Each compound was dissolved in dimethyl sulfuoxide (0.5\% DMSO)

Table 3: Effects of various compounds on the chymotrypsin-like activity of $20 \mathrm{~S}$ rabbit muscle proteasome ${ }^{1}$

\begin{tabular}{|c|c|c|c|c|c|c|c|c|c|c|c|c|c|c|c|c|c|}
\hline & Treatment & & & & & & & & & & & & & & & & \\
\hline \# & $\begin{array}{l}\text { Assay } \\
\text { Mixture }\end{array}$ & $\begin{array}{l}\text { 3. Lacta- } \\
\text { cystin }\end{array}$ & $\begin{array}{l}\text { 4. (-) Corey } \\
\text { Lactone }\end{array}$ & $\begin{array}{l}\text { 5. Oua- } \\
\text { bain }\end{array}$ & $\begin{array}{l}\text { 6. Thios- } \\
\text { trepton }\end{array}$ & $\begin{array}{c}\text { 7. Ri- } \\
\text { fampicin }\end{array}$ & 8. Ampicillin & $\begin{array}{l}\text { 9. } 2-\mathrm{Hy}- \\
\text { drox- } \\
\text { yestradiol }\end{array}$ & $\begin{array}{l}\text { 10. 2-Meth- } \\
\text { oxyestradiol }\end{array}$ & $\begin{array}{l}\text { 11. } 25-\mathrm{Hy}- \\
\text { droxycho- } \\
\text { lesterol }\end{array}$ & $\mu \mathrm{M}$ & $\begin{array}{l}\text { 12. Ace- } \\
\text { tylsalicylic } \\
\text { Acid }\end{array}$ & $\mu \mathrm{M}$ & $\begin{array}{c}13 . \\
\text { Ascarbic } \\
\text { Acid }\end{array}$ & $\begin{array}{l}14 . \\
\text { Nicotinic } \\
\text { Acid }\end{array}$ & $\begin{array}{c}15 . \\
\text { Vitamin } \\
D_{3}\end{array}$ & $\begin{array}{c}16 . \\
\text { Resvera- } \\
\text { trol }\end{array}$ \\
\hline 2 & $\begin{array}{c}\mathrm{A}+0.2 \% \\
\mathrm{DMSO}^{2} \\
=\mathrm{B}\end{array}$ & $\begin{array}{c}1612 \pm 9 \\
(100)^{3}\end{array}$ & $\begin{array}{c}1394 \pm 46 \\
(100)^{3}\end{array}$ & $\begin{array}{c}1572 \pm 21 \\
(100)^{3}\end{array}$ & $\begin{array}{c}1570 \pm 11 \\
(100)^{3}\end{array}$ & $\begin{array}{c}1566 \pm 22 \\
(100)^{3}\end{array}$ & $\begin{array}{c}1462 \pm 18 \\
(100)^{3}\end{array}$ & $\begin{array}{c}1562 \pm 18 \\
(100)^{3}\end{array}$ & $\begin{array}{c}1554 \pm 23 \\
(100)^{3}\end{array}$ & $\begin{array}{c}1564 \pm 23 \\
(100)^{3}\end{array}$ & & $\begin{array}{c}1560 \pm 24 \\
(100)^{3}\end{array}$ & & $\begin{array}{c}1589 \pm 19 \\
(100)^{3}\end{array}$ & $\begin{array}{c}1581 \pm 14 \\
(100)^{3}\end{array}$ & $\begin{array}{c}1572 \\
\pm 24 \\
(100) 3\end{array}$ & $\begin{array}{c}1555 \\
\pm 28 \\
(100) 3\end{array}$ \\
\hline 3 & $\mathrm{~B}+2.5 \mu \mathrm{M}$ & $\begin{array}{c}1543 \pm 12 \\
(96)\end{array}$ & $\begin{array}{c}1598 \pm 55 \\
(115)\end{array}$ & $\begin{array}{c}1662 \pm 12 \\
(106)\end{array}$ & $\begin{array}{c}1317 \pm 8 \\
(84)\end{array}$ & $\begin{array}{c}1186 \pm 15 \\
(76)\end{array}$ & $\begin{array}{c}1740 \pm 22 \\
(119)\end{array}$ & $\begin{array}{c}1432 \pm 13 \\
(92)\end{array}$ & $\begin{array}{c}1461 \pm 17 \\
(94)\end{array}$ & $\begin{array}{c}1370 \pm 26 \\
(94)\end{array}$ & 10 & $\begin{array}{c}1432 \pm 18 \\
(92)\end{array}$ & 2.5 & $\begin{array}{c}1647 \pm 27 \\
(104)\end{array}$ & $\begin{array}{c}1476 \pm 13 \\
(93)\end{array}$ & $\begin{array}{l}1361 \pm \\
30(87)\end{array}$ & $\begin{array}{l}1266 \pm \\
10(81)\end{array}$ \\
\hline 4 & $\mathrm{~B}+5 \mu \mathrm{M}$ & $\begin{array}{c}1343 \pm 11 \\
(83)\end{array}$ & $\begin{array}{c}1900 \pm 64 \\
(136)\end{array}$ & $\begin{array}{c}1775 \pm 13 \\
(113)\end{array}$ & $\begin{array}{c}1275 \pm 9 \\
(81)\end{array}$ & $\begin{array}{c}1017 \pm 18 \\
(65)\end{array}$ & $\begin{array}{c}1845 \pm 11 \\
(126)\end{array}$ & $\begin{array}{c}1254 \pm 30 \\
(80)\end{array}$ & $\begin{array}{c}1262 \pm 37 \\
(81)\end{array}$ & $\begin{array}{c}1274 \pm 23 \\
(82)\end{array}$ & 20 & $\begin{array}{c}1272 \pm 12 \\
(82)\end{array}$ & 5 & $\begin{array}{c}1768 \pm 31 \\
(11)\end{array}$ & $\begin{array}{c}1424 \pm 12 \\
\quad(90)\end{array}$ & $\begin{array}{l}1310 \pm \\
10(83)\end{array}$ & $\begin{array}{l}1164 \pm \\
11(75)\end{array}$ \\
\hline 5 & $\mathrm{~B}+10 \mu \mathrm{M}$ & $\begin{array}{c}1142 \pm 10 \\
(71)\end{array}$ & $\begin{array}{c}2187 \pm 71 \\
(157)\end{array}$ & $\begin{array}{c}1992 \pm 9 \\
(122)\end{array}$ & $\begin{array}{c}1244 \pm 9 \\
(79)\end{array}$ & $\begin{array}{c}1020 \pm 21 \\
(66)\end{array}$ & $\begin{array}{c}1922 \pm 24 \\
(131)\end{array}$ & $\begin{array}{c}1060 \pm 30 \\
(68)\end{array}$ & $\begin{array}{c}1179 \pm 18 \\
(76)\end{array}$ & $\begin{array}{c}1236 \pm 15 \\
(79)\end{array}$ & 40 & $\begin{array}{c}1222 \pm 11 \\
(79)\end{array}$ & 10 & $\begin{array}{c}1957 \pm 38 \\
(123)\end{array}$ & $\begin{array}{c}1384 \pm 15 \\
(71)\end{array}$ & $\begin{array}{l}1273 \pm \\
16(81)\end{array}$ & $\begin{array}{l}1032 \pm \\
12(66)\end{array}$ \\
\hline 6 & $\mathrm{~B}+20 \mu \mathrm{M}$ & $\begin{array}{c}897 \pm 11 \\
(56)\end{array}$ & $\begin{array}{l}2353 \pm \\
83(169)\end{array}$ & $\begin{array}{l}1966 \pm 11 \\
(125)\end{array}$ & $\begin{array}{c}1223 \pm 9 \\
(78)\end{array}$ & $\begin{array}{c}985 \pm 11 \\
(63)\end{array}$ & $\begin{array}{c}1966 \pm 11 \\
(134)\end{array}$ & $\begin{array}{c}870 \pm 15 \\
(56)\end{array}$ & $\begin{array}{l}992 \pm 24 \\
(62)\end{array}$ & $\begin{array}{c}1164 \pm 11 \\
(74)\end{array}$ & 80 & $\begin{array}{c}1035 \pm 19 \\
(66)\end{array}$ & 20 & $\begin{array}{c}2078 \pm 41 \\
(131)\end{array}$ & $\begin{array}{c}1254 \pm 9 \\
(79)\end{array}$ & $\begin{array}{l}1173 \pm \\
18(75)\end{array}$ & $\begin{array}{c}833 \pm 15 \\
(54)\end{array}$ \\
\hline 7 & $\mathrm{~B}+40 \mu \mathrm{M}$ & $\begin{array}{c}777 \pm 12 \\
(48)\end{array}$ & $\begin{array}{c}2645 \pm 84 \\
(190)\end{array}$ & $\begin{array}{c}1995 \pm 11 \\
(127)\end{array}$ & $\begin{array}{l}997 \pm 10 \\
(64)\end{array}$ & $\begin{array}{c}939 \pm 10 \\
(60)\end{array}$ & $\begin{array}{c}2031 \pm 15 \\
(139)\end{array}$ & $\begin{array}{c}634 \pm 11 \\
(41)\end{array}$ & $\begin{array}{c}645 \pm 12 \\
(42)\end{array}$ & $\begin{array}{c}926 \pm 17 \\
(59)\end{array}$ & 160 & $\begin{array}{c}873 \pm 27 \\
(56)\end{array}$ & 40 & $\begin{array}{c}2147 \pm 34 \\
(135)\end{array}$ & $\begin{array}{c}1115 \pm 9 \\
(71)\end{array}$ & $\begin{array}{l}1023 \pm \\
10(65)\end{array}$ & $\begin{array}{c}748 \pm 24 \\
(48)\end{array}$ \\
\hline 8 & $\mathrm{~B}+80 \mu \mathrm{M}$ & $754 \pm 8(47)$ & $\begin{array}{c}2657 \pm 88 \\
(191)\end{array}$ & $\begin{array}{c}2060 \pm 15 \\
(131)\end{array}$ & $\begin{array}{c}972 \pm 7 \\
(62)\end{array}$ & $\begin{array}{c}876 \pm 14 \\
(56)\end{array}$ & $\begin{array}{c}2076 \pm 18 \\
(142)\end{array}$ & $\begin{array}{c}522 \pm 10 \\
(33)\end{array}$ & $\begin{array}{c}431 \pm 19 \\
\quad(28)\end{array}$ & $\begin{array}{l}862 \pm 14 \\
\quad(55)\end{array}$ & 320 & $\begin{array}{c}824 \pm 8 \\
(53)\end{array}$ & 80 & $\begin{array}{c}2210 \pm 39 \\
(139)\end{array}$ & $\begin{array}{c}1027 \pm 7 \\
(65)\end{array}$ & $\begin{array}{c}974 \pm 16 \\
(62)\end{array}$ & $\begin{array}{c}717 \pm 6 \\
(46)\end{array}$ \\
\hline 9 & $\mathrm{~B}+160 \mu \mathrm{M}$ & $\begin{array}{c}515 \pm 12 \\
(32)\end{array}$ & $\begin{array}{c}2719 \pm 98 \\
(195)\end{array}$ & $\begin{array}{c}2171 \pm 8 \\
(138)\end{array}$ & $\begin{array}{c}936 \pm 8 \\
(60)\end{array}$ & $\begin{array}{c}722 \pm 10 \\
(46)\end{array}$ & $2086 \pm 9(143)$ & $\begin{array}{c}450 \pm 16 \\
(29)\end{array}$ & $\begin{array}{l}362 \pm 13 \\
(24)\end{array}$ & $\begin{array}{c}818 \pm 9 \\
(52)\end{array}$ & 640 & $\begin{array}{c}778 \pm 19 \\
(51)\end{array}$ & 160 & $\begin{array}{c}2285 \pm 46 \\
(144)\end{array}$ & $\begin{array}{c}963 \pm 11 \\
(61)\end{array}$ & $\begin{array}{c}922 \pm 10 \\
(59)\end{array}$ & $\begin{array}{c}721 \pm 10 \\
(46)\end{array}$ \\
\hline
\end{tabular}

${ }^{1}$ The thioglycolate-elicited peritoneal macrophages of each mice were adhered to the bottom of $100 \mathrm{~mm}$ tissue culture plates ( $10^{7}$ cells/well in $\left.1.0 \mathrm{ml} \mathrm{media}\right)$ for 4 $\mathrm{h}$, supernatants were removed, and the cells were washed extensively with medium three times. The cells were cultured overnight in fresh medium after the final wash. After overnight inc at $37{ }^{\circ} \mathrm{C}$ in presence of $5 \% \mathrm{CO}_{2}$ for $4 \mathrm{~h}$. The cells were treated with various compounds (100 $\mu \mathrm{l}$, dissolved in $0.2 \% \mathrm{DMSO}$ ) of various concentrations and LPS $(10 \mathrm{ng} / \mathrm{ml} ; 400 \mu \mathrm{l})$ was added to culture solution (LPS final concentration, $10.0 \mathrm{ng} / \mu \mathrm{l})$. The supernatants were collected after $4 \mathrm{~h}$ of incubation at $37{ }^{\circ} \mathrm{C}$ in presence of $5 \% \mathrm{CO}_{2}$ for $4 \mathrm{~h}$. The supernatants were assayed for TNF- $\alpha$ by using ELISA assay kit. Cells viability were $>95 \%$ in all treatments ${ }^{2} \mathrm{DMSO}=$ Dimethyl sulfoxide

${ }^{3}$ Percentage of control values are in parenthesis

${ }^{*}$ Average of triplicate analyses of each sample

Table 4: Effects of selected compounds on the secretion of TNF- $\alpha$ (pg/ml) in LPS-stimulated thioglycolate-elicited peritoneal macrophages obtained from 8-week-old female PPAR-a knockout mice ${ }^{1}$

\section{Evaluation of selected compounds on the secretion of TNF- $\alpha$ in LPS-induced TG-elicited peritoneal mac- rophages obtained from 8-week-old female C57BL/6 (Wild Type), and PPAR- $\alpha$ knockout mice}

The above results prompted us to determine the impact of at least a single dose $(20 \mu \mathrm{M})$ oflactacystin, thiostrepton, 2-hydroxyestradiol, 2-methoxyestradiol, and $40 \mu \mathrm{M}$ for remaining compounds in LPS-induced TG-elicited peritoneal macrophages obtained from 8week-old female C57BL/6 (Wild Type-WT; its control group). Most of the compounds showed significant decreases $33 \%$ to $54 \%$ $(\mathrm{P}<0.01-0.001)$ in the secretion of TNF- $\alpha$ in peritoneal macrophages of C57BL/6 (Wild Type, control group) female mice (Table 5; Figure 2A). 
However, ampicillin and (-) corey lactone showed decreases of $71 \%$ and $76 \%(\mathrm{P}<0.05)$, respectively compared to control (Table 5; Figure 2A). Whereas, using same concentrations of $20 \mu \mathrm{M}$ for lactacystin, thiostrepton, 2-hydroxyestradiol, 2-methoxyestradiol showed significant decreases of $63 \%(\mathrm{P}<0.05), 78 \%(\mathrm{P}<0.05), 56 \%(\mathrm{P}<0.01), 62 \%(\mathrm{P}<0.05)$, respectively compared to respective controls (Table 5, Figure $2 \mathrm{~B}$ ). The $40 \mu \mathrm{M}$ concentration was used for remaining compounds, which resulted significant increases in the secretion of TNF- $\alpha$ with $(-)$ corey lactone $(+90 \%$; $\mathrm{P}<0.001)$, ouabain $(+27 \%$; $\mathrm{P}<0.05)$, ampicillin $(+39 \%$; $\mathrm{P}<0.01)$, and ascorbic acid $(35 \% ; \mathrm{P}<0.01)$ compared to respective controls (Table 5, Figure 2B). These results confirm our earlier findings [1].

\begin{tabular}{|c|c|c|c|c|c|c|c|c|}
\hline & Assay Mixture & $\mu \mathrm{M}$ & $\begin{array}{c}\mathrm{B}=100 \% \\
(\mathrm{C} 57 \mathrm{BL} / 6)\end{array}$ & TNF- $\alpha(p g / \mathrm{ml})$ & Percentages $^{2}$ & $\begin{array}{l}B=100 \% \\
(\text { PPAR- } \alpha)\end{array}$ & $\begin{array}{l}\text { TNF- } \alpha \\
(p g / m l)\end{array}$ & Percentages \\
\hline 1 & $\begin{array}{c}\text { Media }+ \text { cells }+0.2 \% \\
\mathrm{DMSO}^{3}=\mathrm{A}\end{array}$ & & & & & & 0.00 & 0.00 \\
\hline 2 & $A+\operatorname{LPS}(10 n g / \mathrm{ml})=B$ & & $2349 \pm 156 a$ & & 100 & $1551 \pm 56$ & & 100 \\
\hline 3 & B + Lactacystin & 20 & 2245 & $1079 \pm 2712 \mathrm{a},{ }^{\star *}$ & 46 & $1612 \pm 9$ & $856 \pm 11 b,{ }^{*}$ & 63 \\
\hline 4 & B + (-) Corey Lactone & 40 & 2166 & $1822 \pm 171^{*}$ & 76 & $1394 \pm 46$ & $2645 \pm 84^{* * *}$ & 190 \\
\hline 5 & B + Ouabain & 40 & 2346 & $1249 \pm 170^{* *}$ & 53 & $1572 \pm 21$ & $1995 \pm 35^{*}$ & 127 \\
\hline 6 & B + Thiostrepton & 20 & 2092 & $1072 \pm 103^{\star *}$ & 46 & $1570 \pm 11$ & $1223 \pm 9^{*}$ & 78 \\
\hline 7 & B + Rifampicin & 40 & 2536 & $1269 \pm 210^{* *}$ & 54 & $1566 \pm 22$ & $939 \pm 10^{*}$ & 60 \\
\hline 8 & B + Ampicillin & 40 & 2374 & $1666 \pm 91^{*}$ & 71 & $1462 \pm 18$ & $2031 \pm 15^{\star *}$ & 139 \\
\hline 9 & B + 2-Hydroxyestradiol & 20 & 2288 & $1219 \pm 138^{* *}$ & 52 & $1562 \pm 18$ & $870 \pm 15^{* *}$ & 56 \\
\hline 10 & B + 2-Methoxyestradiol & 20 & 2156 & $769 \pm 117^{\star * *}$ & 33 & $1554 \pm 23$ & $992 \pm 24^{*}$ & 62 \\
\hline 11 & B + 25-Hydroxycholestrol & 40 & 2482 & $1105 \pm 171^{\star *}$ & 47 & $1564 \pm 23$ & $926 \pm 17^{*}$ & 59 \\
\hline 12 & B + Acetylsalicylic Acid & 320 & 2566 & $1248 \pm 129^{* *}$ & 53 & $1560 \pm 24$ & $824 \pm 8^{* *}$ & 53 \\
\hline 13 & $\begin{array}{c}\text { B + Ascorbic Acid } \\
(\text { Vitamin C) }\end{array}$ & 40 & 2523 & $1760 \pm 79^{*}$ & 75 & $1589 \pm 19$ & $2147 \pm 34^{\star *}$ & 135 \\
\hline 14 & $\begin{array}{c}\mathrm{B}+\text { Nicotinic Acid } \\
\left(\text { Vitamin } \mathrm{B}_{3}\right)\end{array}$ & 40 & 2371 & $895 \pm 129^{* * *}$ & 38 & $1581 \pm 14$ & $1115 \pm 9^{*}$ & 71 \\
\hline 15 & $\mathrm{~B}+\operatorname{Vitamin} \mathrm{D}_{3}$ & 40 & 2498 & $1211 \pm 67^{*}$ & 52 & $1572 \pm 24$ & $1023 \pm 10^{*}$ & 65 \\
\hline 16 & $\mathrm{~B}+$ trans - Resveratrol & 40 & 2243 & $781 \pm 91^{\star * *}$ & 33 & $1555 \pm 28$ & $748 \pm 24^{\star *}$ & 48 \\
\hline
\end{tabular}

${ }^{1}$ The thioglycolate-elicited peritoneal macrophages of each mice were adhered to the bottom of $100 \mathrm{~mm}$ tissue culture plates $\left(10^{7}\right.$ cells $/$ well in $\left.1.0 \mathrm{ml} \mathrm{medium}\right)$ for $4 \mathrm{~h}$. After $4 \mathrm{~h}$, supernatants were removed, and the cells were washed extensively with medium three times. The cells were cultured overnight in fresh medium after the final wash. After overnight incubation at $37^{\circ} \mathrm{C}$ in presence of $5 \% \mathrm{CO}_{2}$, the cells were treated with various compounds $(100 \mu \mathrm{l}$ dissolved in $0.2 \%$ DMSO) of various concentrations and LPS ( $10 \mu \mathrm{l} \mathrm{of} 1.0 \mu \mathrm{g} / \mathrm{ml}$ stock solution) was added to culture solution (LPS final concentration, $10.0 \mathrm{ng} / \mathrm{ml}$ ). The supernatants were collected after $4 \mathrm{~h}$ of incubation at $37^{\circ} \mathrm{C}$ in presence of $\mathrm{CO}_{2}$ to carry out TNF- $\alpha$ by using ELISA assay kit. Cells viability were $>95 \%$ in all treatments

${ }^{2}$ Percentage of control values are in parenthesis

${ }^{3} \mathrm{DMSO}=$ Dimethyl sulfoxide

${ }^{a}$ Average of fourtein analyses of all samples, and percentage of each compound is calculated against this average

${ }^{\mathrm{b}}$ Average of triplicate analyses of each sample

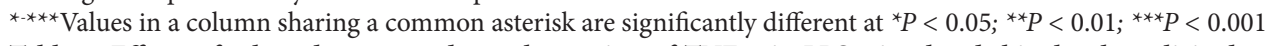

Table 5: Effects of selected compounds on the section of TNF- $\alpha$ in LPS-stimulated thioglycolate-elicited peritoneal macrophages of 8-week-old female C57BL/6 (wild type) and 8-week-old female PPAR- $\alpha$ knockout mice ${ }^{1}$

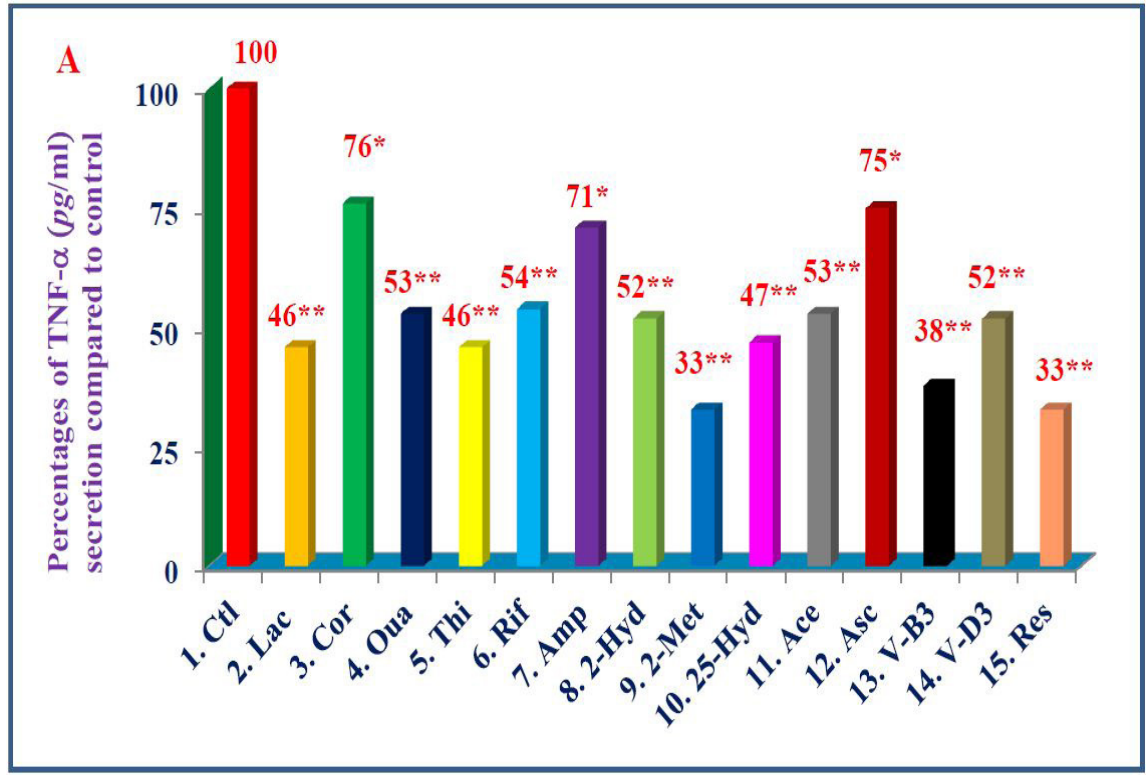




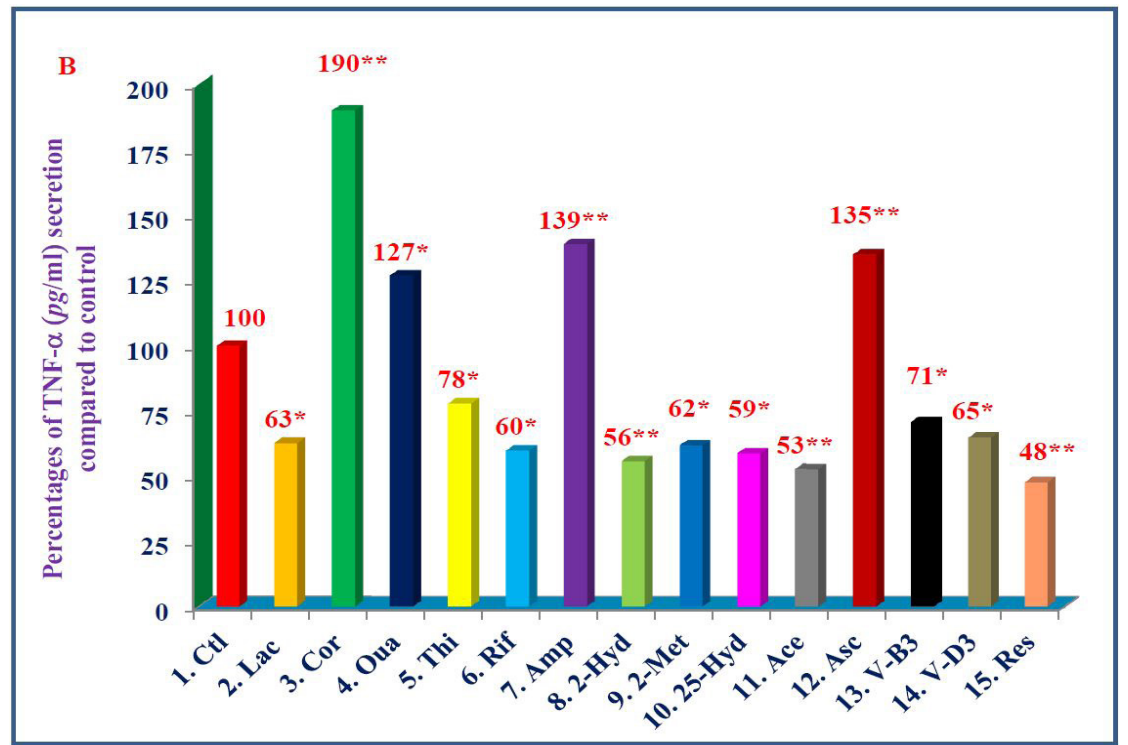

Figure 2A, B: Effects of selected compounds on the secretion of TNF- $\alpha$ in LPS-induced TG- elicited peritoneal macrophages of 8-week-old female C57BL/6 (A), and PPAR- $\alpha$ knockout (B) mice

TG-elicited peritoneal macrophages were prepared from 8-week-old female C57BL/6 (Wild Type; 2A), and PPAR- $\alpha$ knockout (2B) mice as described previously [1]. The macrophages of each mouse were adhered to the bottom of 12 well plates $\left(1 \times 10^{7}\right.$ cells $/$ well in $1 \mathrm{ml}$ media) for $4 \mathrm{~h}$. After 4 h, supernatants were removed, and the cells were washed extensively with medium three times. The cells were cultured overnight in fresh medium after final wash. After overnight incubation at $37{ }^{\circ} \mathrm{C}$ in presence of $5 \% \mathrm{CO}_{2}$, the cells were treated with various compounds ( $100 \mu \mathrm{l}$ dissolved in $0.2 \%$ DMSO) of different concentrations of 3 . lactacystin $(20 \mu \mathrm{M}) ; 4$. (-) corey lactone $(40 \mu \mathrm{M}) ; 5$. ouabain $(40 \mu \mathrm{M}) ; 6$. thiostrepton $(20 \mu \mathrm{M}) ; 7$. rifampicin $(40 \mu \mathrm{M}) ; 8$. ampicillin $(40 \mu \mathrm{M}) ; 9.2$-hydroxyestradiol $(20 \mu \mathrm{M}) ;$ 10. 2-methoxyestradiol $(20 \mu \mathrm{M}) ; 11$. 25-hydroxycholesterol $(40 \mu \mathrm{M}) ; 12$. acetylsalicylic acid $(320 \mu \mathrm{M}) ; 13$. ascorbic acid (40 $\mu \mathrm{M}) ; 14$. nicotinic acid (40 $\mu \mathrm{M}) ; 15$. vitamin $\mathrm{D}_{3}(40 \mu \mathrm{M})$; 16. trans-resveratrol $(40 \mu \mathrm{M})$ plus LPS $(10 \mathrm{ng} / \mathrm{ml})$ of each treatment. The supernatants were collected after $4 \mathrm{~h}$ of incubation at $37{ }^{\circ} \mathrm{C}$ in presence of $\mathrm{CO}_{2}$ to carry out TNF- $\alpha$ by using ELISA assay kit. Data are average of triplicate analyses of each sample as \pm SD (standard deviation). Percentage values of each treatment compared to control are at the top of the column. Values in a column not sharing a common asterisk are significantly different at ${ }^{\star} \mathrm{P}<$ $0.05 ;{ }^{* *} \mathrm{P}<0.01 ;{ }^{* *} \mathrm{P}<0.001$

\section{Evaluation of selected compounds on production of nitric oxide (NO) in LPS-induced TG- elicited perito- neal macrophages obtained from 8-week-old female PPAR- $\alpha$ knockout mice}

Similarly, we have determined the effects of same 14 compounds (Table 3) on production of nitric oxide (NO) using concentrations between $20 \mu \mathrm{M}-40 \mu \mathrm{M}$ for all compounds except acetylsalicylic acid $(320 \mu \mathrm{M})$ in LPS-induced TG-elicited peritoneal macrophages obtained from 8-week-old female PPAR- $\alpha$ knockout mice. There were dose-dependent decreases in production of nitric oxide by all compounds in this system (Table 6). The significant $(\mathrm{P}<0.01-0.001)$ reduction in production of nitric oxide was observed with (-) corey lactone (50\%), rifampicin (47\%), 2-hydroxyestradiol (58\%), 2-methoxyestradiol (55\%), 25-hydroxycholesterol (63\%), nicotinic acid (45\%), and trans-resveratrol (50\%) compared to controls (Table 6, Figure 3). The treatment with acetylsalicylic acid (aspirin) showed 23\% $(\mathrm{P}<0.01)$ decrease, even with a very high dose $(320 \mu \mathrm{M})$ compared to control (Table 6, Figure 3). Perhaps, that is why high dose of $>200 \mathrm{mg} / \mathrm{d}$ aspirin are prescribed most of the time, because acidic $\mathrm{pH}$ may have inhibited the activity.

\begin{tabular}{|c|c|c|c|c|c|}
\hline & Assay Mixture & $\mu \mathrm{M}$ & $\begin{array}{c}\text { Control } \\
(\mu \mathrm{M})\end{array}$ & $\begin{array}{l}\text { Nitric oxide } \\
(\mathrm{NO} ; \mu \mathrm{M})\end{array}$ & Percentages $^{2}$ \\
\hline 1 & $\begin{array}{c}\text { Media }+ \text { cells }+0.2 \% \mathrm{DMSO}^{3} \\
=\mathrm{A}\end{array}$ & & & 0.00 & 0.00 \\
\hline 2 & $A+\operatorname{LPS}(10 \mathrm{ng} / \mathrm{ml})=B$ & & $23.07 \pm 2.63^{*}$ & & 100 \\
\hline 3 & B + Lactacystin & 20 & & $15.22 \pm 1.97^{*}$ & 66 \\
\hline 4 & $\mathrm{~B}+(-)$ Corey Lactone & 40 & & $11.44 \pm 0.92^{* *}$ & 50 \\
\hline 5 & B + Ouabain & 40 & & $13.12 \pm 1.87^{*}$ & 57 \\
\hline 6 & B + Thiostrepton & 20 & & $15.86 \pm 1.67^{\star}$ & 69 \\
\hline 7 & B + Rifampicin & 40 & & $12.25 \pm 1.71^{* *}$ & 53 \\
\hline 8 & B + Ampicillin & 40 & & $14.12 \pm 1.61^{*}$ & 61 \\
\hline 9 & B + 2-Hydroxyestradiol & 20 & & $9.61 \pm 1.67^{* *}$ & 42 \\
\hline 10 & B + 2-Methoxyestradiol & 20 & & $10.41 \pm 1.15^{\star *}$ & 45 \\
\hline 11 & B + 25-Hydroxycholestrol & 40 & & $8.43 \pm 0.79^{* *}$ & 37 \\
\hline 12 & B + Acetylsalicylic Acid & 320 & & $17.73 \pm 0.85^{*}$ & 77 \\
\hline 13 & B + Ascorbic Acid (Vitamin C) & 40 & & $15.53 \pm 0.61^{*}$ & 66 \\
\hline 14 & B + Nicotinic Acid (Vitamin $B_{3}$ ) & 40 & & $12.67 \pm 0.55^{\star}$ & 55 \\
\hline
\end{tabular}




\begin{tabular}{|c|c|c|c|c|c|}
\hline & Assay Mixture & $\boldsymbol{\mu M}$ & $\begin{array}{c}\text { Control } \\
(\boldsymbol{\mu M})\end{array}$ & $\begin{array}{c}\text { Nitic oxide (NO; } \\
\boldsymbol{\mu M})\end{array}$ & Percentages $^{2}$ \\
\hline 15 & $\mathrm{~B}+$ Vitamind $\mathrm{D}_{3}$ & 40 & & $13.41 \pm 1.18^{*}$ & 58 \\
\hline 16 & $\mathrm{~B}+$ trans - Resveratrol & 40 & & $11.44 \pm 1.12^{\star *}$ & 50 \\
\hline
\end{tabular}

${ }^{1}$ The thioglycolate-elicited peritoneal macrophages $\left(1 \mathrm{x} 10^{7}\right.$ cells/well in $500.0 \mu \mathrm{l}$ medium) of each mice were adhered to the bottom of $100 \mathrm{~mm}$ tissue culture plates for $4 \mathrm{~h}$. After $4 \mathrm{~h}$, supernatants were removed, and the cells were washed with medium three times. The cells were treated with various concentrations of each compound ( $100 \mu \mathrm{l}$ dissolved in $0.2 \%$ DMSO), and induced with LPS (10 ng /well; $400 \mu \mathrm{l})$. The assay mixtures were incubated at $37{ }^{\circ} \mathrm{C}$ in presence of $5 \% \mathrm{CO}_{2}$ for $36 \mathrm{~h}$. The supernatants were assayed for the production of nitric oxide by measuring the amount of nitrite using Griess reagent. Data are presented as the percent of nitric oxide (NO) levels compared to control

${ }^{2}$ Percentage of control values are in parenthesis

${ }^{3} \mathrm{DMSO}=$ Dimethyl sulfoxide. ${ }^{\star}$ Average of triplicate analyses of each sample

${ }^{*}{ }^{* *}$ Values in a column sharing a common asterisk are significantly different at ${ }^{\star} P<0.01 ;{ }^{* *} P<0.001$

Table 6: Effects of selected compounds on the production of nitric oxide (NO; $\mu \mathrm{M})$ in LPS-induced thioglycolate-elicited peritoneal macrophages of 8-week-old female PPAR- $\alpha$ knockout mice ${ }^{1}$

\section{Evaluation of selected compounds on time-dependent secretion of TNF- $\alpha$ in LPS-induced TG-elicited peritoneal macrophages of 8-week-old female PPAR- $\alpha$ knockout mice}

The earlier experiments showed dose-dependent increases with treatment of (-) corey lactone, ouabain, ampicillin and ascorbic acid in secretion of TNF- $\alpha$ and decreases in induction of nitric oxide with all remaining 10 compounds (Tables 5,6) tested in TGelicited peritoneal macrophages derived from PPAR- $\alpha$ knockout female mice. Therefore, we thought of value to estimate timedependent effects of these compounds (Tables 5,6) for secretion of TNF- $\alpha$ by using identical conditions using same macrophages, as described in previous paragraphs. In the present experiment, we have checked the effects of these compounds (Tables 5,6) after incubation of 1 h. $2 \mathrm{~h}$, and $3 \mathrm{~h}$ only, because estimation of TNF-a was carried out earlier after incubations of $4 \mathrm{~h}$. The treatments with (-) corey lactone, ouabain, ampicillin and ascorbic acid showed time-dependent increases in secretion of TNF- $\alpha$ varies between $104 \%-130 \%$ as shown in Table 7. The rest of the ten compounds showed time-dependent significant decreases in TNF- $\alpha$ secretion between $1 \mathrm{~h}--23 \%$ to $3 \mathrm{~h}--43 \%$ (Table 7 ). The maximum decrease was observed with thiostrepton (43\%) and 2-hydroxyestradiol (41\%) among these compounds (Table 7). When these increases or decreases were compared values of secretion TNF- $\alpha$ with $4 \mathrm{~h}$ earlier incubation (Table 5) resulted slightly higher increases (127\% to 190\%), and decrease were closely similar to $3 \mathrm{~h}$ of incubation, indicated that $4 \mathrm{~h}$ of incubation time used earlier experiments was correct for these biomarkers for all these compounds.

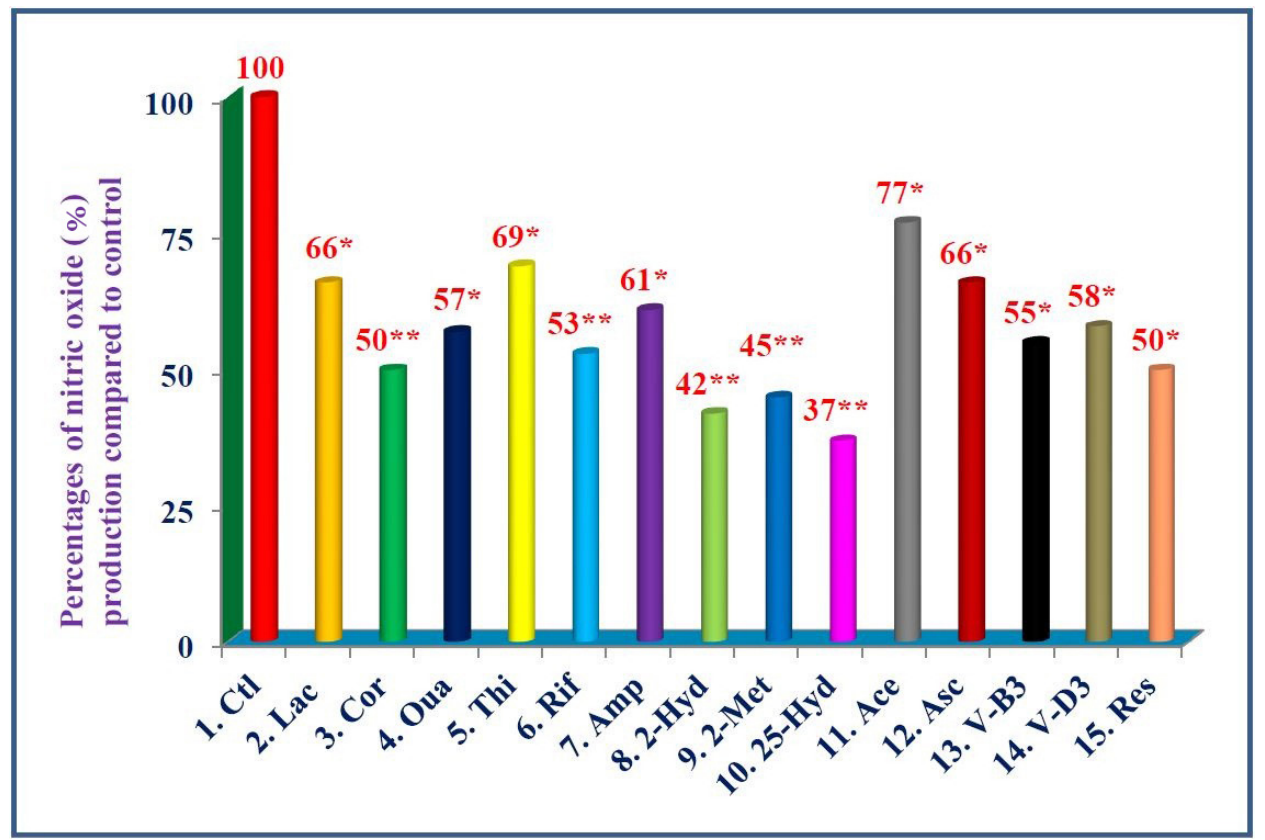

Figure 3: Effects of selected compounds on the productions of nitric oxide (NO) in LPS- induced TG-elicited peritoneal macrophages of 8-week-old female PPAR- $\alpha$ knockout mice

TG-elicited peritoneal macrophages were prepared from 8 -week-old female PPAR- $\alpha$ knockout mice as described previously [1]. The macrophages (1 x10 ${ }^{6}$ cells /well in $1.0 \mathrm{ml}$ medium) were adhered to the bottom of $100 \mathrm{~mm}$ tissue culture plate for $4 \mathrm{~h}$ and then supernatants were removed, and cells were washed with medium three times. The cells were cultured overnight in fresh medium after the final wash. After overnight incubation at $37{ }^{\circ} \mathrm{C}$ in presence of $5 \% \mathrm{CO}_{2}$, the cells were treated with different concentrations of following compounds (100 $\mu \mathrm{l}$ in $0.2 \%$ DMSO) of 3 . lactacystin (20 $\mu \mathrm{M}) ; 4$. (-) corey lactone ( $40 \mu \mathrm{M}) ; 5$. ouabain $(40 \mu \mathrm{M}) ; 6$. thiostrepton $(20 \mu \mathrm{M}) ; 7$. rifampicin $(40 \mu \mathrm{M}) ; 8$. ampicillin $(40 \mu \mathrm{M}) ; 9$. 2-hydroxyestradiol $(20 \mu \mathrm{M}) ; 10.2$-methoxyestradiol $(20 \mu \mathrm{M}) ; 11$. 25-hydroxycholesterol $(40 \mu \mathrm{M}) ; 12$. acetylsalicylic acid $(320 \mu \mathrm{M}) ; 13$. ascorbic acid $(40 \mu \mathrm{M}) ; 14$. nicotinic acid $(40 \mu \mathrm{M}) ; 15$. vitamin $\mathrm{D}_{3}(40 \mu \mathrm{M}) ; 16$. transresveratrol $(40 \mu \mathrm{M})$ plus LPS $(10 \mathrm{ng} / \mathrm{ml})$ of each treatment. The assay mixtures were incubated at $37^{\circ} \mathrm{C}$ in presence of $5 \% \mathrm{CO}_{2}$ for $36 \mathrm{~h}$. After $36 \mathrm{~h}$ of incubation, the supernatants were assayed for the production of nitric oxide by measuring the amount of nitrite using Griess reagent. Data are average of triplicate analyses of each sample as \pm SD (standard deviation). Percentage values of each treatment compared to control are at the top of the column. Values in a column not sharing a common asterisk are significantly different at ${ }^{*} \mathrm{P}<0.01 ;{ }^{*} \mathrm{P}<0.001$ 


\begin{tabular}{|c|c|c|c|c|c|c|c|c|}
\hline$\#$ & Assay Mixture & hour. & TNF- $\alpha(p g / \mathrm{ml})$ & $\#$ & & $\mu \mathrm{M}$ & hour. & TNF- $\alpha(p g / \mathrm{ml})$ \\
\hline 1 & Media + cells $=\mathrm{A}$ & & 0 & 1 & Media + cells $=\mathrm{A}$ & & & 0 \\
\hline 2 & $\mathrm{~A}+0.2 \% \mathrm{DMSO}^{2}=\mathrm{B}$ & & 1845 & 2 & $\mathrm{~A}+0.2 \% \mathrm{DMSO}^{2}=\mathrm{B}$ & & & 1845 \\
\hline 3 & $A+\operatorname{LPS}(10.0 n g / \mathrm{ml})=C$ & & $\begin{array}{c}1674-1473 \\
(100)^{3}\end{array}$ & 3 & $\mathrm{~A}+\mathrm{LPS}(10 \mathrm{ng} / \mathrm{ml})=\mathrm{C}$ & & & $\begin{array}{c}1674-1473 \\
(100)^{3}\end{array}$ \\
\hline \multirow[t]{4}{*}{4} & C + Lactacystin & 0 & $1674(100)$ & 11 & $\mathrm{C}+2$-Methoxyestradiol & 20 & 0 & $1567(100)$ \\
\hline & C + Lactacystin & 1 & $1490(89)$ & & $\mathrm{C}+2$-Methoxyestradiol & & 1 & $1332(85)$ \\
\hline & $C+$ Lactacystin & 2 & $1389(83)$ & & $\mathrm{C}+2$-Methoxyestradiol & & 2 & $1175(75)$ \\
\hline & C + Lactacystin & 3 & $1172(70)$ & & $\mathrm{C}+2$-Methoxyestradiol & & 3 & $987(63)$ \\
\hline \multirow[t]{4}{*}{5} & $\mathrm{C}+(-)$ Corey Lactone & 0 & $1537(100)$ & 12 & $C+25$-Hydroxycholesterol & 40 & 0 & $1588(100)$ \\
\hline & $\mathrm{C}+(-)$ Corey Lactone & 1 & $1668(109)$ & & C +25-Hydroxycholesterol & & 1 & 1207 \\
\hline & $\mathrm{C}+(-)$ Corey Lactone & 2 & $1818(118)$ & & $C+25$-Hydroxycholesterol & & 2 & 826 \\
\hline & $C+(-)$ Corey Lactone & 3 & $1969(128)$ & & $C+25$-Hydroxycholesterol & & 3 & 667 \\
\hline \multirow[t]{4}{*}{6} & $\mathrm{C}+$ Ouabain & 0 & $1486(100)$ & 13 & $C+$ Acetylsalicylic Acid & 320 & 0 & $1574(100)$ \\
\hline & $\mathrm{C}+$ Ouabain & 1 & $1650(111)$ & & C + Acetylsalicylic Acid & & 1 & $1255(79)$ \\
\hline & $\mathrm{C}+$ Ouabain & 2 & $1768(119)$ & & $C+$ Acetylsalicylic Acid & & 2 & $1191(75)$ \\
\hline & $\mathrm{C}+$ Ouabain & 3 & $1843(124)$ & & $C+$ Acetylsalicylic Acid & & 3 & $1048(66)$ \\
\hline \multirow[t]{4}{*}{7} & C + Thiostrepton & 0 & $1473(100)$ & 14 & $C+$ Ascorbic Acid (Vitamin C) & 40 & 0 & $1582(100)$ \\
\hline & $\mathrm{C}+$ Thiostrepton & 1 & $1208(82)$ & & $\mathrm{C}+$ Ascorbic Acid (Vitamin C) & & 1 & $1692(107)$ \\
\hline & $\mathrm{C}+$ Thiostrepton & 2 & $1134(77)$ & & $\mathrm{C}+$ Ascorbic Acid (Vitamin C) & & 2 & $1772(112)$ \\
\hline & $\mathrm{C}+$ Thiostrepton & 3 & $840(57)$ & & $\mathrm{C}+$ Ascorbic Acid (Vitamin C) & & 3 & $2051(130)$ \\
\hline \multirow[t]{4}{*}{8} & $\mathrm{C}+$ Rifampicin & 0 & $1473(100)$ & 15 & $\mathrm{C}+$ Nicotinic Acid $\left(\right.$ Vitamin $\left.\mathrm{B}_{3}\right)$ & 40 & 0 & $1586(100)$ \\
\hline & $\mathrm{C}+$ Rifampicin & 1 & $1252(85)$ & & $C+$ Nicotinic Acid (Vitamin $\left.B_{3}\right)$ & & 1 & $1396(88)$ \\
\hline & $\mathrm{C}+$ Rifampicin & 2 & $1105(75)$ & & $\mathrm{C}+$ Nicotinic Acid $\left(\right.$ Vitamin $\left.\mathrm{B}_{3}\right)$ & & 2 & $1316(83)$ \\
\hline & C + Rifampicin & 3 & $884(60)$ & & $C+$ Nicotinic Acid $\left(\right.$ Vitamin $\left.B_{3}\right)$ & & 3 & $1158(73)$ \\
\hline \multirow[t]{4}{*}{9} & $\mathrm{C}+$ Ampicillin & 0 & $1486(100)$ & 16 & $\mathrm{C}+$ Vitamin $\mathrm{D}_{3}$ & 40 & 0 & $1573(100)$ \\
\hline & $\mathrm{C}+$ Ampicillin & 1 & $1548(104)$ & & $\mathrm{C}+$ Vitamin $\mathrm{D}_{3}$ & & 1 & $1321(84)$ \\
\hline & $\mathrm{C}+$ Ampicillin & 2 & $1670(112)$ & & $\mathrm{C}+$ Vitamin $\mathrm{D}_{3}$ & & 2 & $1195(76)$ \\
\hline & $\mathrm{C}+$ Ampicillin & 3 & $1892(127)$ & & $\mathrm{C}+$ Vitamin $\mathrm{D}_{3}$ & & 3 & $1085(69)$ \\
\hline \multirow[t]{4}{*}{10} & C + 2-Hydroxyestradiol & 0 & $1496(100)$ & 17 & $\mathrm{C}+$ trans -Resveratrol & 40 & 0 & $15.82(100)$ \\
\hline & $\mathrm{C}+2$-Hydroxyestradiol & 1 & $1154(77)$ & & $\mathrm{C}+$ trans - Resveratrol & & 1 & $14.04(89)$ \\
\hline & C + 2-Hydroxyestradiol & 2 & $1139(76)$ & & $\mathrm{C}+$ trans - Resveratrol & & 2 & $13.14(83)$ \\
\hline & $C+2$-Hydroxyestradiol & 3 & $884(59)$ & & $\mathrm{C}+$ trans - Resveratrol & & 3 & $12.78(81)$ \\
\hline
\end{tabular}

${ }^{1}$ The thioglycolate-elicited peritoneal macrophages of each mice were adhered to the bottom of $100 \mathrm{~mm}$ tissue culture plates $\left(10^{7}\right.$ cells/well in $\left.1.0 \mathrm{ml} \mathrm{medium}\right)$ for $4 \mathrm{~h}$. After $4 \mathrm{~h}$, supernatants were removed, and the cells were washed extensively with medium three times. The cells were cultured overnight in fresh medium after the final wash. After overnight incubation at $37^{\circ} \mathrm{C}$ in $\mathrm{CO}_{2}$, the cells were treated with various compounds (dissolved in $\left.0.2 \% \mathrm{DMSO}\right)^{2}$ of various concentrations and LPS ( $10 \mu \mathrm{l} \mathrm{of} 1.0 \mu \mathrm{g} / \mathrm{ml}$ stock solution) was added to culture solution (LPS final concentration, $10.0 \mathrm{ng} / \mathrm{ml}$ ). The supernatants were collected after $4 \mathrm{~h}$ of incubation at $-37^{\circ} \mathrm{C}$ in $\mathrm{CO}_{2}$ to carry out TNF- $\alpha$ ELISA assay kit; Cells viability were $>95 \%$ in all treatments ${ }^{2} \mathrm{DMSO}=$ Dimethyl sulfoxide

${ }^{3}$ Percentage of control values are in parenthesis

Table 7: Effects of selected compounds on time-dependent secretion of TNF- $\alpha$ in LPS-induced thioglycolated-elicited peritoneal macrophages of 8-weekold female PPAR- $a$ knockout mice ${ }^{1}$ 


\section{Evaluation of selected compounds on gene expression of TNF- $\alpha$, IL-1 $\beta$, IL-6 and iNOS in LPS-induced TG-elicited peritoneal macrophages obtained from 8-week-old female PPAR- $\alpha$ knockout mice}

The experiments described above demonstrated that out of fourteen compounds, (-) corey lactone, ouabain, ampicillin and ascorbic acid showed increases in the secretion of TNF- $\alpha$ and remaining compounds resulted decreases in LPS-induced TGelicited peritoneal macrophages from PPAR- $\alpha$ knockout female mice. In contrast, all these compounds showed decreases in nitric oxide production in LPS-induced TG-elicited peritoneal macrophages derived from PPAR-a knockout female mice. In order to determine whether these changes resulted from alterations in transcription of the relevant genes, we measured the effect of all these compounds on mRNA levels for various cytokines (TNF- $\alpha$, IL-1 $\beta$, IL-6) and iNOS enzyme activity in LPS-induced. TG-elicited peritoneal macrophages from 8-week-old PPAR- $\alpha$ knockout female mice. The concentrations (in $\mu \mathrm{M}$ ) of each compound and conditions were similar to those used in earlier experiments, and macrophages were treated with selected compounds plus LPS (10 ng/well), and incubated for $4 \mathrm{~h}$. Total cellular RNA was then extracted by using RNAeasy mini kit and reverse-transcribed, and gene analyses were carried out by RT-PCR.

The (-) corey lactone, ouabain, ampicillin and ascorbic acid treatment significantly up- regulated in mRNAs expression of TNF- $\alpha$ $(31 \%, 36 \%, 60 \%, 46 \%)$, respectively compared to control in LPS-induced TG-elicited peritoneal macrophages derived from PPAR- $\alpha$ knockout female mice (Table 8, Figure 4). On the other hand, treatments with rest of the compounds markedly down-regulated gene expression of TNF- $\alpha$ and other biomarkers. Each of the above compounds significantly down-regulated gene expression of IL-1 1 (5\% - 69\%), IL-6 (4\% - 37\%) and iNOS activity (11\% - 33\%) compared to control (Table 8, Figures 5-7). Lactacystin, a positive inhibitor tested in macrophages obtained from PPAR- $\alpha$ knockout mice markedly down-regulated mRNA expression for TNF- $\alpha$ (31\%), IL-1 $\beta$ (69\%), IL-6 (84\%), and iNOS activity (39\%) compared to their respective controls (Table 8, Figures 4-7). The acetylsalicylic acid treatment of these macrophages down-regulated gene expressions less profoundly compared to other compounds of these four biomarkers $10 \%, 13 \%, 9 \%$, and $11 \%$, respectively compared to controls (Table 8 ). In summary, these results of mRNA expression studies were generally consistent with those of TNF- $\alpha$ secretion and NO production, though the enhanced secretion of TNF- $\alpha$ by LPS-induced macrophages from PPAR- $\alpha$ knockout mice treated with (-) corey lactone, ouabain, ampicillin and ascorbic acid was not explained by a corresponding increase in mRNA levels.

\begin{tabular}{|c|c|c|c|c|c|c|}
\hline & \multirow[t]{2}{*}{ Assay Mixture } & \multirow[t]{2}{*}{$\mu \mathrm{M}$} & \multicolumn{3}{|c|}{$\begin{array}{l}\text { Ratios of cytokines/ } \beta \text {-actin of relative } \\
\text { optical density of various genes }\end{array}$} & \multirow[b]{2}{*}{ iNOS } \\
\hline & & & TNF- $\alpha$ & IL-1 $\beta$ & IL-6 & \\
\hline 1 & $\begin{array}{c}\text { Media + cells + } \\
0.2 \% \mathrm{DMSO}^{2}=\mathrm{A}\end{array}$ & & 4.81 & 2.23 & 2.11 & 1.54 \\
\hline 2 & $\begin{array}{c}\mathrm{A}+\mathrm{LPS}(10.0 n g / \mathrm{ml}) \\
=\mathrm{B}\end{array}$ & & $4.72(100)^{3}$ & $2.15(100)^{3}$ & $1.95(100)^{3}$ & $1.37(100)^{3}$ \\
\hline 3 & B + Lactacystin & 20 & $3.24(69)$ & $0.67(31)$ & $0.31(16)$ & $0.84(61)$ \\
\hline 4 & B + (-) Corey Lactone & 40 & $6.18(131)$ & $1.27(59)$ & $1.27(65)$ & $0.98(72)$ \\
\hline 5 & B + Ouabain & 40 & $6.43(136)$ & $1.43(67)$ & $1.42(73)$ & $1.12(82)$ \\
\hline 6 & B + Thiostrepton & 20 & $2.12(45)$ & $1.47(68)$ & $1.23(63)$ & $0.96(70)$ \\
\hline 7 & B + Rifampicin & 40 & $2.22(47)$ & $1.29(60)$ & $1.31(67)$ & $0.94(69)$ \\
\hline 8 & $\mathrm{~B}+$ Ampicillin & 40 & $7.56(160)$ & $2.01(93)$ & $1.85(95)$ & $1.15(84)$ \\
\hline 9 & B + 2-Hydroxyestradiol & 20 & $1.98(42)$ & $1.54(72)$ & $1.74(89)$ & $1.21(88)$ \\
\hline 10 & B + 2-Methoxyestradiol & 20 & $2.11(45)$ & $1.59(74)$ & $1.79(92)$ & $0.97(71)$ \\
\hline 11 & $\begin{array}{l}\text { B + 25-Hydroxycho- } \\
\text { lesterol }\end{array}$ & 40 & $1.67(35)$ & $1.28(60)$ & $1.87(96)$ & $0.92(67)$ \\
\hline 12 & B + Acetylsalicylic Acid & 320 & $4.23(90)$ & $1.88(87)$ & $1.78(91)$ & $1.22(89)$ \\
\hline 13 & $\begin{array}{l}\text { B + Ascorbic Acid } \\
\quad(\text { Vitamin } C)\end{array}$ & 40 & $6.87(146)$ & $1.72(80)$ & $1.56(80)$ & $1.21(88)$ \\
\hline 14 & $\begin{array}{l}\text { B + Nicotinic Acid (Vi- } \\
\left.\operatorname{tamin} B_{3}\right)\end{array}$ & 40 & $2.68(57)$ & $2.03(94)$ & $1.44(74)$ & $1.22(89)$ \\
\hline 15 & $\mathrm{~B}+\operatorname{Vitamin} \mathrm{D}_{3}$ & 40 & $2.77(59)$ & $2.05(95)$ & $1.34(69)$ & $1.19(87)$ \\
\hline 16 & $\mathrm{~B}+$ trans - Resveratrol & 40 & $3.47(74)$ & $1.67(78)$ & $1.26(65)$ & $1.16(85)$ \\
\hline
\end{tabular}

${ }^{1}$ The thioglycolate-elicited peritoneal macrophages of each mice were adhered to the bottom of 12 well plate $\left(10^{6} \mathrm{cells} /\right.$ well in $1.0 \mathrm{ml}$ medium $)$ for $2 \mathrm{~h}$. After $2 \mathrm{~h}$, the cells were treated with $100 \mu \mathrm{l}$ of various compounds (dissolved in 0.2\% DMSO) and LPS (10.0 ng /well in $400 \mu \mathrm{l}$ ), and incubated at room temperature for 4 h. Total RNAs were extracted from each cell pellet, and real-time PCR was conducted to quantitate TNF- $\alpha$, IL-1 $\beta$, IL- 6 , and iNOS genes from each experiment. Data are presented as the percent of mRNAs of the genes analysed compared to respective controls

${ }^{2} \mathrm{DMSO}=$ Dimethyl sulfoxide

${ }^{3}$ Percentage of control values are in parenthesis

Table 8: Effects of selected compounds on gene expression of TNF- $\alpha$, IL-1 $\beta$, IL-6 and iNOS in 8-week-old female PPAR- $\alpha$ knockout Mice ${ }^{1}$ 


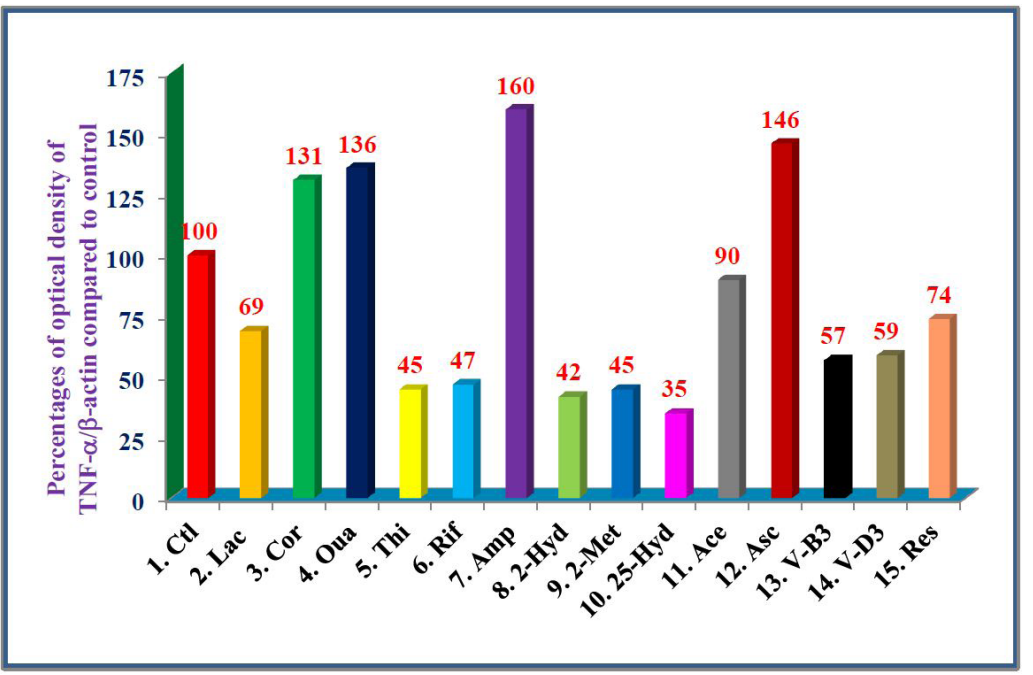

Figure 4: The effect of different compounds on gene expression of TNF- $\alpha$ in LPS-induced TG-elicited peritoneal macrophages from 8-week-old female PPAR- $\alpha$ knockout mice.

TG-elicited peritoneal macrophages were prepared from 8-week-old female PPAR- $\alpha$ knockout mice, adhered to the bottom of 12 well plates $\left(1 \times 10^{7}\right.$ cells/well in $1 \mathrm{ml}$ medium) for $2 \mathrm{~h}$ and then treated with $100 \mu \mathrm{l}$ (dissolved in $0.2 \% \mathrm{DMSO}$ ) of various concentrations of 3. lactacystin (20 $\mu \mathrm{M}) ; 4$. (-) corey lactone (40 $\mu \mathrm{M})$; 5. ouabain $(40 \mu \mathrm{M})$; 6. thiostrepton $(20 \mu \mathrm{M})$; 7. rifampicin $(40 \mu \mathrm{M}) ; 8$. ampicillin $(40 \mu \mathrm{M}) ; 9$. 2-hydroxyestradiol $(20 \mu \mathrm{M})$; 10. 2-methoxyestradiol $(20 \mu \mathrm{M})$; 11. 25-hydroxycholesterol $(40 \mu \mathrm{M}) ; 12$. acetylsalicylic acid $(320 \mu \mathrm{M}) ; 13$. ascorbic acid $(40 \mu \mathrm{M}) ; 14$. nicotinic acid $(40 \mu \mathrm{M}) ; 15$. vitamin $\mathrm{D}_{3}(40 \mu \mathrm{M})$; 16 . transresveratrol $(40 \mu \mathrm{M})$ plus LPS $(10 \mathrm{ng} / \mathrm{ml})$ of each treatment. The assay mixtures were incubated at room temperature for $4 \mathrm{~h}$. Total cellular RNA was extracted using RNAeasy mini kit to quantitate gene expression of TNF- $\alpha$. Data expressed as \pm SD (standard deviation) as percentage of control ratio, based on medium plus macrophages plus LPS $10 \mathrm{ng} /$ well in $0.2 \%$ dimethyl sulfoxide (DMSO) for each of the gene analyzed compared to control.

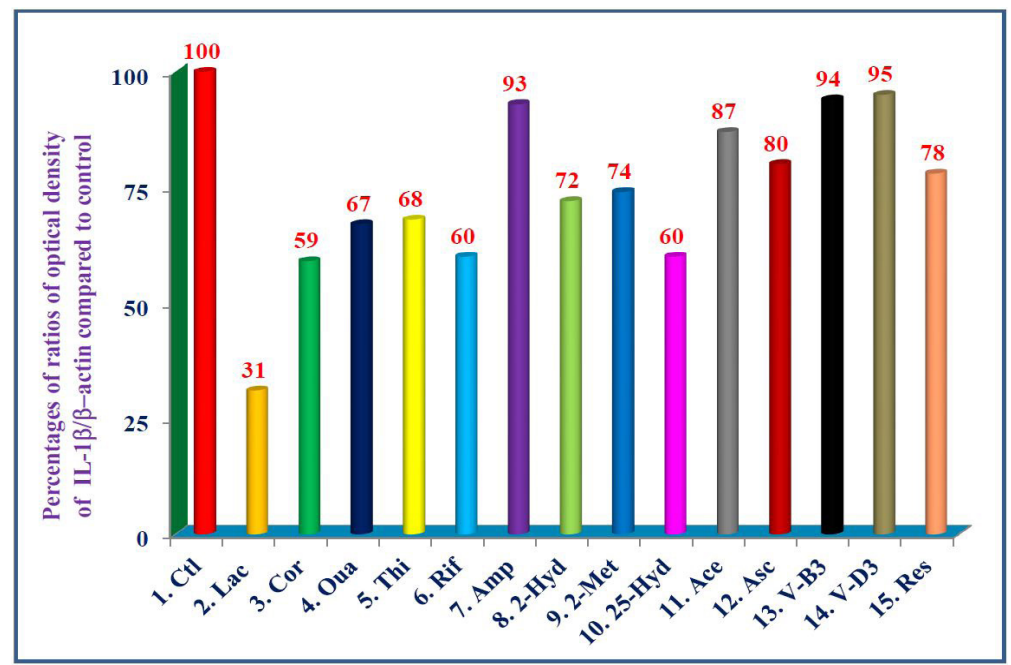

Figure 5: The effect of different compounds on gene expression of interleukin-1 $\beta$ (IL-1 $\beta$ ) in LPS-induced TG-elicited peritoneal macrophages from 8-week-old female PPAR- $\alpha$ knockout mice: The procedure to quantitate gene expression of IL-1 $\beta$ was exactly same as described in Figure 4

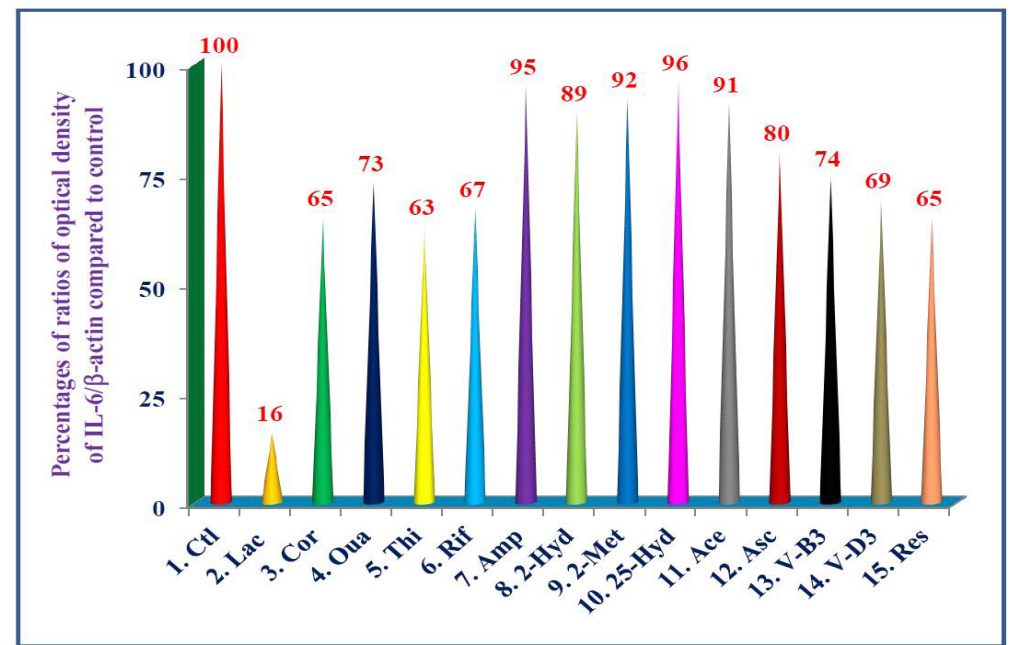

Figure 6: The effect of different compounds on gene expression of interleukin-6 (IL-6) in LPS-induced TG-elicited peritoneal macrophages from 8-week-old female PPAR- $a$ knockout mice: The procedure to quantitate gene expression of IL- 6 was exactly same as described in Figure 4 


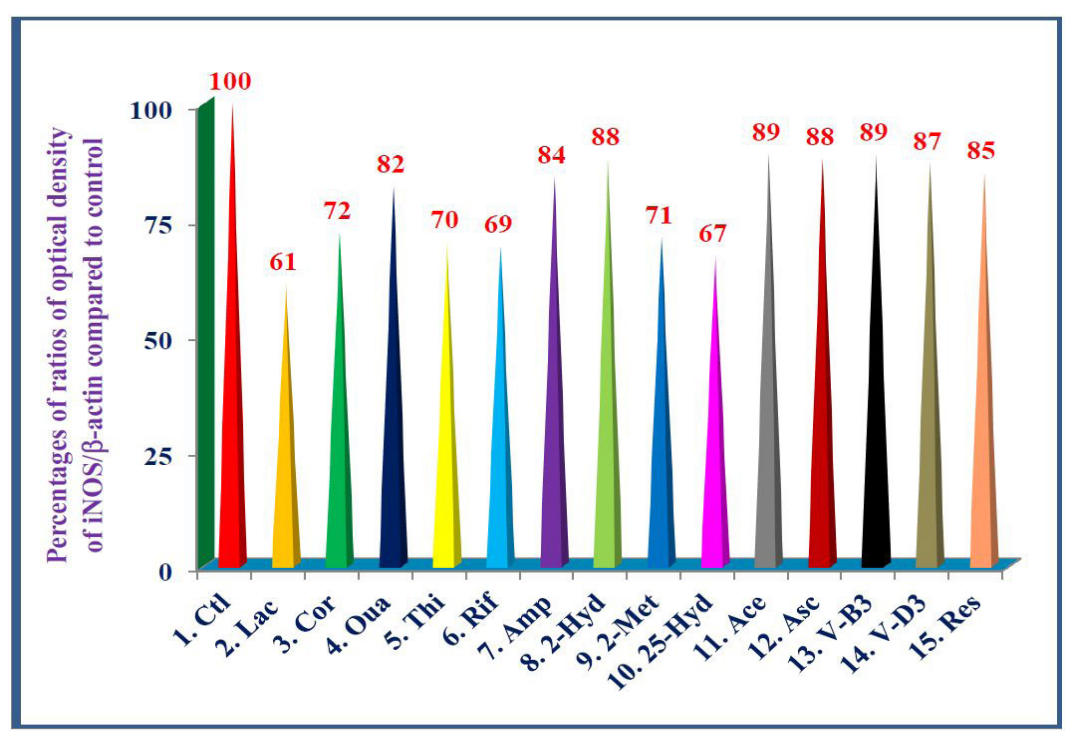

Figure 7: The effect of different compounds on gene expression of iNOS enzyme in LPS- induced TG-elicited peritoneal macrophages from 8-week-old female PPAR- $\alpha$ knockout mice: The procedure to quantitate gene expression of iNOS was exactly same as described in Figure 4.

\section{Discussion}

The primary objective in the present study was to evaluate anti-inflammatory and pro- inflammatory properties of several compounds in macrophages derived from PPAR- $\alpha$ knockout mice. As a result of these studies we have identified several compounds that could potentially decrease or increase levels of secretion of inflammatory cytokines and production of nitric oxide that may contribute to treatment of several human diseases. First, we have demonstrated that thiostrepton, rifampicin (broad spectrum antibiotics), 2-hydroxyestradiol, 2-methoxyestradiol, 25-hydroxycholesterol, nicotinic acid, vitamin $\mathrm{D}_{3}$, and trans-resveratrol caused significant decreases in chymotrypsin-like activity of 20S rabbit muscle proteasomes, and followed by significant increases with (-) corey lactone, ouabain, ampicillin, ascorbic acid, codeine, amiloride-HCL chymotrypsin-like activity of $20 \mathrm{~S}$ rabbit muscle proteasomes compared to respective control groups. These results indicated that there are two distinct sets of compounds, one set of compounds causes decrease, and second set of compounds resulted in increase in $20 \mathrm{~S}$ activity. This conclusion was further supported in the secretion TNF- $\alpha$ by both two set of compounds in LPS-induced TG-elicited peritoneal macrophages derived from PPAR- $\alpha$ knockout mice as observed for 20S activity. In marked contrast to observations with TNF- $\alpha$, all compounds suppressed nitric oxide (NO) production in LPS-induced TG-elicited peritoneal macrophages from PPAR- $\alpha$ knockout mice. The effect of both sets of compounds on gene expression of TNF- $\alpha$, IL-1 $\beta$, IL- 6 , and iNOS in LPS-induced macrophages from PPAR- $\alpha$ knockout mice were down-regulated or up-regulated, which were generally consistent with at the protein levels of secretion of TNF- $\alpha$ and production of nitric oxide (NO).

As described earlier that PPAR- $\alpha$ knockout mice have exaggerated inflammatory responses to a variety of stimuli, because activation of PPAR- $\alpha$ leads to anti-inflammatory effects [15]. The mechanisms leading to these exaggerated inflammatory responses are believed to be at least partially attributable to increased NF- $\kappa B$ activity $[15,16]$. Consequently, one would expect TNF- $\alpha$ secretion by LPS-induced macrophages from PPAR- $\alpha$ knockout mice to be highly up-regulated and relatively resistant to inhibition by some of these compounds that degrade $\mathrm{p}-\mathrm{I} \kappa \mathrm{B}$, and decrease NF- $\kappa \mathrm{B}$ activity. Therefore, (-) corey lactone, ouabain, ampicillin, ascorbic acid, codeine, amiloride-HCL failed to suppress TNF- $\alpha$ secretion by LPS-induced macrophages from PPAR- $\alpha$ knockout mice.

Apart from present results, importance of other inflammatory biological functions of these compounds were realized by checking the published reports of other investigators [17-40]. Lactacystin is a potent proteasome inhibitor and played important role in ubiquitin-proteasome pathway in various cellular inflammatory processes, such as cell cycle, apoptosis, and the degradation of regulatory or membrane proteins [17]. (-) Corey lactone is a hydroxyl-lactone intermediate for the synthesis of prostaglandins and prostaglandin analogs, and ouabain plays an active role in the transport of $\mathrm{Na}^{+}-\mathrm{K}^{+}$-ATPase in the brain, and also involved in the regulation of several inflammatory cell functions (proliferation, hypertrophy, apoptosis, mobility, and metabolism) [18,19]. Ouabain is endogenously produced in mammals and circulates in plasma as a hormone in normal condition and disease. It induces $\mathrm{Na}^{+}-\mathrm{K}^{+}$-ATPase signaling in cytogenesis of autosomal dominant polycystic kidney disease, hypertension, and also provides cardioprotection against stressful stimuli such as ischemia [20,21].

Thiostrepton is a natural potent proteasome inhibitor, and induces apoptotic cell death in human cancer cells. It also induces oxidative and proteotoxic stress by up-regulating the stress-related genes as well as endoplasmic reticulum stress genes [22]. Whereas, rifampicin is one of most potent broad spectrum anti-inflammatory antibiotics against bacterial pathogens used to treat tuberculosis by inhibiting the bacterial RNA polymerase by blocking the pathway of elongating RNA in humans [23]. Ampicillin is a potent anti-inflammatory antibiotic to treat respiratory tract infections, urinary tract infections, meningitis, salmonellosis, and endocarditis [24]. 
2-Hydroxyestradiol plays an important inflammatory role in breast carcinogenesis by increased cell proliferation and formation of reactive oxygen species, which is due to increase deoxyribonucleic acid mutations [25]. 2-Methoxyestradiol, an estrogen hormone metabolite is also a potent cancer chemotherapeutic agent, and causes induction of apoptosis in transformed and exhibit an antiproliferative effect on tumor growth. Moreover, its anticancer activity has been attributed to its anti-tubulin, anti-angiogenic, pro-apoptotic and ROS induction properties [25,26]. 25-Hydroxycholesterol plays important role in maintenance of cholesterol homeostasis for supplying tissues with proper amount of cholesterol and prevent accumulation that may affect health of the individual [27]. Moreover, 25-hydroxycholesterol and one of its metabolites are also involved in regulation of immunity. Therefore, 25-hydroxycholesterol may be much more important as regulator of immunity than as a regulator of cholesterol metabolism in humans [27].

Acetylsalicylic acid (aspirin) is most widely used anti-inflammatory medicine "over-the- counter" in world [28]. Aspirin has been used as analgesic (pain reliever) by blocking the action of COX enzyme, which produces prostaglandins needed for pain response; therefore, it is used to treat headaches, aches and pains [28]. Its anti-inflammatory property makes it an effective medicine to treat arthritis and other rheumatologic diseases $[28,29]$. It also reduces the risk of stroke and heart attacks by reducing the platelet aggregation [30]. It has been established that platelet aggregates adhere to walls of blood vessels and block blood flow resulting in heart attack. Aspirin is very effective in thinning the blood, thus preventing stroke in humans [30]. It has a very important property of antipyretic (fever-reducing) by acting on hypothalamus (a small gland in the brain) that helps to regulate the body temperature [31]. Aspirin has been used as inflammatory agent to treat various types of cancers, diabetes, and Alzheimer disease (dementia) in humans $[32,33]$.

Ascorbic acid is involved many cellular reactions. Its mechanism of action is involved in synthesis of collagen, and its major function seems to keep prosthetic metal ions in their reduced form [34]. It has a very good anti-oxidant property, and protects several tissues from harmful oxidative products by keeping certain enzymes in their required reduced form [34]. Nicotinic acid is synthesized from essential amino acid (tryptophan) in plants and animals. The important role of this vitamin is to lower cholesterol and particularly triglycerides level in blood [35]. It also plays important roles in diabetes, arthritis, and blood pressure. This vitamin is required for healthy state of nervous system, and is essential for synthesis of sex hormones (estrogen, progesterone, testosterone), andocortisone, thyroxin and insulin [35]. There are two major vitamin D derivatives, ergocalciferol ( $\left.\mathrm{D}_{2}\right)$, and cholecalciferol $\left(\mathrm{D}_{3}\right)$ found naturally in fish-liver oil, egg yolks, and liver and vitamin $\mathrm{D}_{3}$ is fat soluble.. These vitamins are photosynthesized in skin of vertebrates by solar ultraviolet radiation, and transported to liver, where it is converted to 25 -hydroxyvitamin $\mathrm{D}$ [36,37]. This 25-hydroxyvitamin D is converted into 1,25 - hydroxyvitamin D in the kidneys, and its biological function is due to 1,25-hydroxyvitamin D in humans [38]. The production of 25-hydroxyvitamin D and 1,25-hydroxyvitamin D is tightly regulated in the liver and kidneys. The activity of vitamin D-hydroxylase is down-regulated by vitamin D and its metabolites, thus limiting its increase in the circulating concentration of 25- hydroxyvitamin D following intakes (fortified milk or food products) or production of vitamin D after exposure to sunlight [38]. trans-Resveratrol is a potent inflammatory agent, found in red grapes, and well known for its chemo-preventive efficacy against several types cancers, and also involves in several other cellular processes as described in the present study. All these published studies clearly indicate the importance of these compounds in the area of cardiovascular, diabetes, and various types of cancers [39,40].

In short, our present results demonstrated that thiostrepton, rifamcipin, 2- hydroxyestradiol, 2-methoxyestradiol, 25-hydroxycholesterol, nicotinic acid, vitamin $\mathrm{D}_{3}$, and resveratrol are potent inhibitors for the inhibition of production of nitric oxide tested in vitro. These compounds also down-regulated inflammatory cytokines and gene expression of TNF- $\alpha$, IL- $1 \beta$, IL-6, and iNOS enzyme activity after response to LPS. The possible mechanism as reported earlier that these compounds blocked LPS-induced

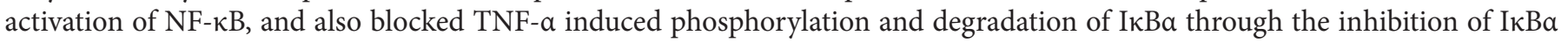
kinase activation [41].

\section{Conclusion}

The present study describes that there are distinct two sets of compounds tested in the study, first set consisted of (-) corey lactone, ouabain, ampicillin, ascorbic acid, codeine, amiloride-HCL, which are potent activators of chymotrypsin-like activity of 20S rabbit muscle proteasomes, and second set consisted of thiostrepton, rifamcipin (broad spectrum antibiotics), 2-hydroxyestradiol, 2-methoxyestradiol, 25-hydroxycholesterol, nicotinic acid, vitamin $\mathrm{D}_{3}$, and resveratrol are potent inhibitors of chymotrypsin-like activity of $20 \mathrm{~S}$ rabbit muscle proteasome. These two sets also have similar effects on levels of secretion of TNF- $\alpha$ tested in vitro using LPS-induced TG-elicited peritoneal macrophages of PPAR- $\alpha$ knockout mice. However, all compounds also blocked production of nitric oxide due to activation of NF- $\kappa \mathrm{B}$, and degradation of $\mathrm{p}-\mathrm{I} \kappa \mathrm{B}$ as described earlier [1]. Similarly, these compounds either up-regulate/down-regulated gene expression of secretion of TNF- $\alpha$ and down-regulated production of nitric oxide, IL-1 $\beta$, IL- 6 and iNOS tested in LPS-induced TG-elicited peritoneal macrophages of PPAR-a knockout mice. The results of present study have provided two sets of compounds, pro-inflammatory (might be useful for treatment of other diseases based on no inflammation), and anti-inflammatory (might be useful for control of diabetes and cardiovascular disease).

\section{Acknowledgements}

We thank Dr. Naheed Dilshad, MBBS, MPH (Pakistan Ordinance Factory Hospital, Wah Cantt, Rawalpindi, Pakistan) for search of 
references on Internet. The study was supported in part by Advanced Medical Research, Madison, Wisconsin and NIH funds R01 GM50870, 5R01GM 102631 and 3R01GM631S1. The study was carried out under a FDA approved IND number 36906.

\section{References}

1. Qureshi AA, Tan X, Reis JC, Badr MZ, Papasian CJ, et al. (2011) Suppression of nitric oxide induction and pro-inflammatory cytokines by novel proteasomes inhibitors in various experimental models. Lipids Health Dis 11: 177.

2. Qureshi N, Takayama K, Mascagni P, Honovich J, Wong R, et al. (1988) Complete structural determination of lipopolysaccharide obtained from deep rough mutant of Escherichia coli. Purification by high performance liquid chromatography and direct analysis by plasma desorption mass spectrometry. J Biol Chem 263: 11971-76.

3. Shulman AI, Mangelsdorf DJ (2005) Retinoid X receptor heterodimers in the metabolic syndrome. N Engl J Med 353: 604-15.

4. Qureshi N, Vogel SN, Way CV III, Papasian CJ, Qureshi AA, et al. (2005) The Proteasome: A central regulator of inflammation and macrophage function. Immunol Res 31: 243-60.

5. Shen J, Gao JJ, Zhang G, Tan X, Morrison DC, et al. (2006) Proteasome inhibitor, lactacystin blocks CpG DNA- and peptidoglycan induced inflammatory genes, cytokines and mitogen-activated protein kinase in macrophages. Shock. 25: 594-99.

6. Qureshi AA, Tan X, Reis JC, Badr MZ, Papasian CJ, et al. (2011) Inhibition of nitric oxide in LPS-stimulated macrophages of young and senescent mice by tocotrienol and quercetin. Lipids Health Dis 10: 239.

7. Chung HY, Cesari M, Anton S, Marzetti E, Giovannini S, et al. (2009) Molecular inflammation: underpinnings of ageing and age-related diseases. Ageing Res Rev 8: $18-30$

8. Nair MP, Mahajan S, Reynolds JL, Aalinkeel R, Nair H, et al. (2006) The flavonoid quercetin inhibits pro-inflammatry cytokine (tumor necrosis factor alpha) gene expression in normal peripheral blood mononuclear cells via modulation of NF-kB system. Clinical and Vaccine Immunology 13: 319-28.

9. Nam N (2006) Naturally-occurring NF-кB inhibitors. Mini Rev Med Chem 6: 945-51.

10. Stuehr DJ, Nathan CF (1989) Nitric oxide: a macrophage product responsible for cytostatic and respiratory inhibition in tumor target cells. J Exp Med 169: $1543-55$

11. Knott AB, Bossy-Wetzel E (2010) Impact of nitric oxide on metabolism in health and age-related disease. Diabetes Obes Metab 12: 126-33.

12. Qureshi N, Perera PY, Splitter G, Morrison DC, Vogel SN, et al. (2003) The Proteasome as a LPS-binding protein in macrophages: Toxic lipopolysaccharide activates the proteasome complex. J Immunol 171: 1515-25.

13. Reis J, Tan X, Yang R, Qureshi AA, Papasian CJ, et al. (2008) A combination of proteasome inhibitors and antibiotics prevents lethality in a septic shock model. Innate Immunity 14: 319-29.

14. Chomczynski P, Saachi N (1987) Single step method of RNA isolation by acid guanidinium thiocyanate-phenol-chloroform extraction. Ana Biochem 162: 156-59.

15. Babaev VR, Ishiguro H, Ding L, Yancey PG, Dove DE, et al. (2007) Macrophage expression of peroxisome proliferator-activated receptor- $\alpha$ reduces atherosclerosis in low-density lipoprotein receptor-deficient mice. Circulation 116:1404-12.

16. Bensinger SJ, Tontonoz P (2008) Integration of metabolism and inflammation by lipid- activated nuclear receptors. Nature 454: 470-77.

17. Tomoda H, Omura S (2015) Lactacystin, a proteasome inhibitor: discovery and its application in cell biology. Rinso Byori 63: 612-22.

18. Chulz S, Hotling S (2015) The use of the lactone motif in chemical communication. Nat Prod Rep 32: 1042-66.

19. Blanco G, Wallace DP (2013) Novel role of ouabain as a cytogenic factor in autosomal dominant polycyclic kidney disease. Am J Physiol Renal Physiol. 305: F797-12

20. Hamlyn JM and Blaustein MP (2014) Salt sensitivity, endogenous ouabain and hypertension. Curr Opin Nephrol Hypertens 22: 51-8.

21. Fuerstenwerth H (2014) Ouabain-the key to cardio-protection. Am J Ther 21: 395-402.

22. Sandu C, Ngounou Wetie AG. Darie CC, Steller H (2014) Thiostrepton, a natural compound that triggers heat shock response and apoptosis in human cancer cells: a proteomics investigation. Adv Exp Med Biol 806: 443-51.

23. Cambell EA, Korzheva N, Mustaev A, Murakami K, Nair S, Goldfarb A, Darst SA (2001) Structural mechanism of rifampicin inhibition of bacterial RNA polymerase. Cell. 104: 901-12.

24. Yano T, Kagamiyama H (2001) Directed evolution of ampicillin-resistant activity from a functionally unrelated DNA fragment: A laboratory model of molecular evolution. PNAS 98: 903-7.

25. Joubert A, Van Zyl H, Laurens J, Lottering ML (2009) C2- and C4-postion 17beta-estradiol metabolites and their relation to breast cancer. Biocell 33: 137-40.

26. Kumar BS, Raghuvanshi DS, Hasanain M, Alam S, Sarkar J, et al. (2016) Recent advances in chemistry and pharmacology of 2-methoxyestradiol: An anticancer investigational drug. Steroids. 110: 9-34.

27. Diczfalusy U (2013) On the formation and possible biological role of 25- hydroxycholesterol. Biochimie. 95: 455-60.

28. Buchanan MR (2006) Biological basis and clinical implication of acetylsalicylic acid resistance. Can J Cardiol. 22: 149-51.

29. Saxena A, Kumar RK, Gera RP, Radhakrishan S, Mishra S, Ahmed Z (2008) Consensus guidelines on pediatric acute rheumatic fever and rheumatic heart disease. Indian Pediatr 45: 565-73.

30. Gasparyan AY, Watson T, Lip GY (2008) The role of aspirin in cardiovascular prevention: Implication of aspirin resistance. J Am Coll Cardiol 51: 1829-43.

31. Hersh E, Moor PA, Ross GL (2000) Over the counter analgesics and antipyretics: A critical assessment. Clin Ther 22: 500-48.

32. Rothwell PM, Wilson M, Price JF, Belch JFF, Meade TW, et al. (2012) Effect of daily aspirin on risk of cancer metastasis: a study of incident cancers during randomized controlled trials. Lancet 379: 1591-601.

33. Bosetti C, Rosato V, Gallus S, Cuzick J, La Vecchia C (2012) Aspirin and cancer risk: a quantitative review to 2011. Ann Oncol 23: 1403-15.

34. Lohmann W (1984) Structure of ascorbic acid and its biological function. VI. Its importance for the $\mathrm{Na}^{+} / \mathrm{K}^{+}$-transport. Biophys Struct Mech 10: 201-10.

35. Kamanna VS, Kashyap ML (2008) Mechanism of action of niacin. Am J Cardiol 101: 20B-6B.

36. Fieser LF, Fieser M (1959) Vitamin D. In Steroids. First edition. New York: Reinhold Publishing Corporation. 90-168. 
37. DeLuca HF (1986) The metabolism, physiology, and function of vitamin D. M Nijhoff Publisher 1-68.

38. DeLuca HF (1988) The vitamin D story: a collaborative story of basic science and clinical medicine. FASEB J 2: 224-36.

39. Reichel H, Koeffler HP, Norman AW (1989) The role of vitamin D endocrine system in health and disease. N Engl J Med 320: 980-91.

40. Tsai HY, Ho CT, Chen YK (2017) Biological actions and molecular effects of resveratrol, pterostilbene, and 3-hydroxypterostilbene. J Food and Drug Anal 25: 134-47.

41. Ahn KS, Sethi G, Krishnan K, Aggarwal BB (2007) $\gamma$-Tocotrienol inhibits nuclear factor- $\kappa B$ signaling pathway through inhibition of receptor- interacting protein and TAK1 leading to suppression of anti-apoptotic gene products and potentiation of apoptosis. J Biol Chem 282: 809-20.

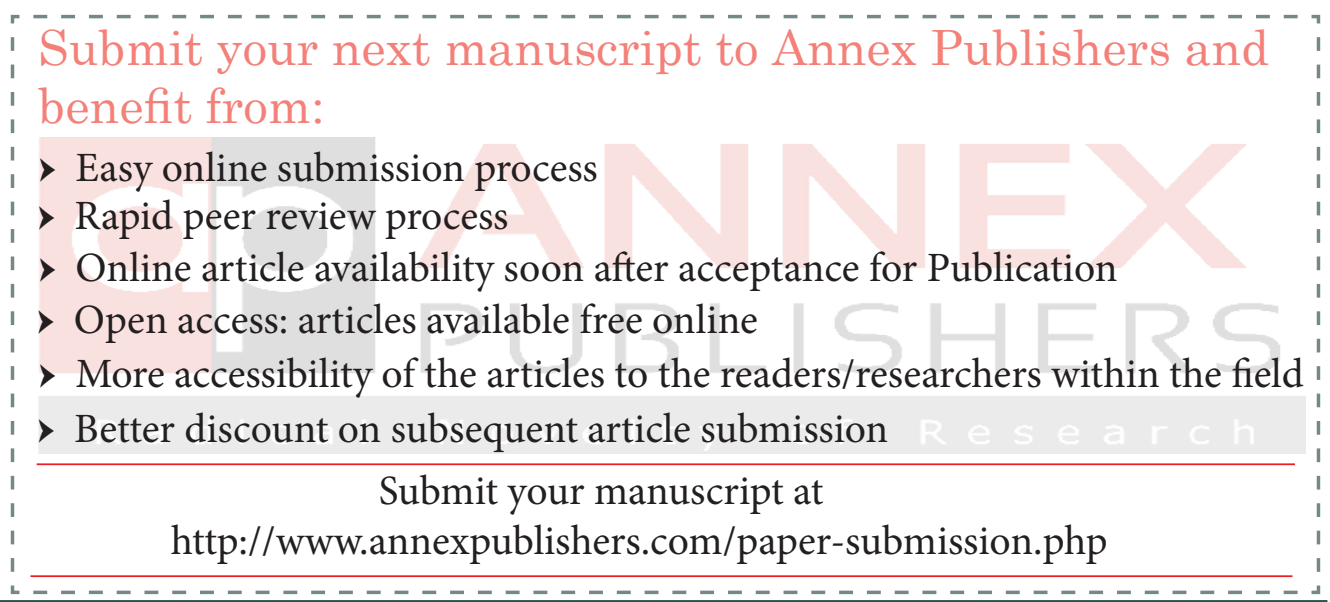

